\title{
LARP6C regulates selective mRNA translation to promote pollen tube guidance in Arabidopsis thaliana.
}

Elodie Billey ${ }^{1,2,3^{*}}$, Said Hafidh ${ }^{5^{*}}$, Isabel Cruz-Gallardo ${ }^{6,7}$, Celso G. Litholdo $\mathrm{Jr}^{1,2}$, Viviane Jean ${ }^{1,2}$, Marie-Christine Carpentier ${ }^{1,2}$, Claire Picart ${ }^{1,2}$, Katarina Kulichova ${ }^{5}$, David Honys ${ }^{5}$, Maria R. Conte ${ }^{6}$, Jean-Marc Deragon ${ }^{1,2,4 @}$ and Cécile BousquetAntonelli1,2,@

\section{Authors Affiliations:}

${ }^{1}$ CNRS LGDP-UMR5096, 58 Av. Paul Alduy 66860 PERPIGNAN, FRANCE.

2 Université de Perpignan Via Domitia, LGDP-UMR5096, 58 Av. Paul Alduy 66860 PERPIGNAN, FRANCE.

3 Elodie Billey present address: TOTAL RC, 2 place Jean Millier- Arche Nord Coupole/Regnault, 92078 Paris La Défense Cedex, FRANCE

${ }^{4}$ Institut Universitaire de France, 1 rue Descartes, 75231 Paris Cedex 5 France

${ }^{5}$ Laboratory of Pollen Biology, Institute of Experimental Botany Czech Academy of Science, Rozvojovà 263, 16502 PRAGUE 6, CZECH REPUBLIC.

${ }^{6}$ Randall Centre for Cell and Molecular Biophysics, King's College London, New Hunt's House, Guy's Campus, LONDON SE1 1UL, UK.

7 Isabel Cruz-Gallardo present address: Department fo Chemistry, King's College London, Britannia House, 7 Trinity St, London SE1 1DB, UK

\section{Corresponding authors:}

cecile.antonelli@univ-perp.fr

hafidh@ueb.cas.cz

jean-marc.deragon@univ-perp.fr

*:These authors contributed equally to the work

Short title: AtLARP6C in pollen tube guidance

The authors responsible for distribution of materials integral to the findings presented in this article in accordance with the policy described in the instruction for Authors (www.plantcell.org) are : Dr. C. Bousquet-Antonelli (cecile.antonelli@univ-perp.fr), Said Hafidh (hafidh@ueb.cas.cz- and Pr. JM Deragon (jean-marc.deragon@univperp.fr). 


\section{ABSTRACT}

In angiosperms, non-motile sperm cells are delivered to the ovules for fertilization via a guided growth of the pollen tube. RNA binding proteins are key regulators that control mRNA fate post-transcriptionally and thus essential for normal cell function. But very little is known on the mechanistic bases of mRNA regulations and trans-acting factors governing male-female signalling. Here we demonstrate that the evolutionarily conserved RNA binding protein LARP6C is necessary for pollen tube guidance and fertilization. larp6c loss-of-function mutants exhibit male induced fertility defects as mutant pollen tubes frequently are unable to find ovules for fertilization. In mature pollen, LARP6C localises with a pollen specific poly(A) binding protein to cytoplasmic foci likely containing mRNPs. With RNA immunoprecipitation and sequencing, we demonstrate that LARP6C is associated in vivo with mRNAs required for pollen tube guidance or polarized cell growth. We further demonstrate using in vitro and in planta transient assays that LARP6C binds 5'-UTR box motifs to orchestrate the balance between translation, decay and storage of its mRNA targets. We propose a model where LARP6C maintains its mRNA target in translationally silent state likely to promote localized translation and guided pollen tube growth upon paracrine signalling. 


\section{INTRODUCTION}

The La Motif (LaM) is a structured RNA binding domain shared by hundreds of eukaryote proteins called La and Related Proteins (LARPs) which classify into five distinct subfamilies, amongst which the LARP6 group (Bousquet-Antonelli and Deragon 2009). In most LARPs, an RNA Recognition Motif (RRM1) is found immediately after the LaM, and forms a bipartite RNA binding unit called the La-module (Maraia et al. 2017). While the LaM is usually very well conserved between subfamilies, the RRM1s are specific to each subgroup (Bousquet-Antonelli and Deragon 2009; Maraia et al. 2017). Members of the LARP6 subfamily are found in Stramenopiles, Chlorophytes, plants, invertebrates and vertebrates (BousquetAntonelli and Deragon 2009; Merret et al. 2013b) and are characterized by the Lamodule and by a short conserved motif known as LSA at the C-terminus (LaM and $\underline{\text { }} 1$ Associated) (Bousquet-Antonelli and Deragon 2009) that mediates protein-protein interactions (Weng et al. 2009; Vukmirovic et al. 2013; Cai et al. 2010b; Manojlovic et al. 2017). In mammals, LARP6 regulates the synthesis of type I collagen, an heterotrimer composed of two $\alpha 1$ and one $\alpha 2$ subunits whose assembly and correct secretion are dependent upon the coordinate translation of their transcripts at the endoplasmic reticulum (ER) (Zhang and Stefanovic 2016). The expression of type I collagen is primarily regulated by LARP6 binding through its La-module, to a stem loop in their 5'-UTRs (Cai et al. 2010a; Martino et al. 2015). LARP6 either stores or stabilizes the collagen mRNA transcripts in a translationally silent form by tethering them to vimentin filaments where they can further be activated for translation or subjected to decay (Challa and Stefanovic 2011). Alternatively LARP6 docks the mRNAs to nonmuscle myosin filaments (Cai et al. 2010b; Manojlovic et al. 2017) and either recruits, via its LSA, STRAP (Serine Threonine kinase Receptor Associated Protein) (Vukmirovic et al. 2013) or the RNA helicase A (RHA) (Manojlovic et al. 2017), which respectively function as repressor and activator of translation. Through this mechanism, LARP6 orchestrates the coordinated translation of $\alpha 1$ and $\alpha 2$ collagen transcripts at the ER.

While LARP6 proteins are generally encoded by a single gene, vascular plant proteins are encoded by 3 to 6 genes and are of three evolutionary types, we named $6 \mathrm{~A}, 6 \mathrm{~B}$ and 6C (Merret et al. 2013b). Whilst members of the 6A subgroup most closely resemble LARP6 from other eukaryotes, B- and C-type orthologs carry, in addition to 
a reorganized La-module, a PABP-interacting motif 2 (PAM2). Arabidopsis thaliana has three AtLARP6 genes, one from each type that we will refer to as LARP6A, B and C throughout this manuscript.

We previously demonstrated that LARP6C directly associates with the poly $(A)$ binding protein (PABP) in plant, consistent with the presence of a PAM2 motif, and that its Lamodule binds to oligo $\left(\mathrm{U}_{20}\right)$ homopolymers in vitro. In addition, in onion epidermis, LARP6C accumulates in the cytoplasm, the nucleoplasm and the nucleolus. Strikingly, LARP6C redistributes to stress granules (SGs) upon hypoxia associating herein with mRNP aggregates (Merret et al. 2013b). These data support the view that Arabidopsis LARP6C is an RNA binding protein likely to intervene in mRNA post-transcriptional regulations.

In flowering plants, the haploid male gamete (pollen grain) consists of a vegetative cell that encases two sperm cells, which are connected (through a so called cytoplasmic connexion) with the vegetative cell nucleus to form the male germ unit. A characteristic feature of angiosperm sexual reproduction is that sperm cells are non motile and must be carried simultaneously to the ovule by a pollen tube (Johnson and Preuss 2002). Upon adhesion to the stigma, the male vegetative cell hydrates, germinates and grows into a tube that delivers the two sperm cells to the ovule. The pollen tube is a greatly polarized and fast tip-growing cell which growth directionality is determined by various perceived cues. It is firstly guided in the gynoeceum by the pistil female sporophytic tissue (preovular guidance) then, upon exit from the transmitting tract, it navigates in gametophytic tissues guided by ovule-emitted signals (ovular guidance). Ovular cues guide the pollen tube alongside the funiculus to the micropyle. Upon reaching the ovule, the pollen tube stops growing and bursts to free the sperm cells that will fuse respectively with the egg cell (to form the embryo) and the central cell (to form the endosperm), hence completing the fecundation process (Higashiyama and Takeuchi 2015). In the pollen tube, upon reception and transduction of extracellular female signals, a complex cellular process targets the cell wall synthesis machinery at the site of perception, hence permitting the delivery of macromolecules at site of extension and the directional growth of the tube. This requires tip-localized receptors, flow of ions, intracellular trafficking of signalling molecules and proteins, but also vesicular trafficking, cytoskeleton-dependent transport and novel formation of cell wall through exocytosis (Feng et al. 2018; Hafidh et al. 2014; Higashiyama and Takeuchi 2015). The pollen tube growth also requires changes in the pattern of gene expression that at 
least in part rely on post-transcriptional mechanisms. Several data indeed suggest that all mRNAs required for germination and pollen tube growth are already present in the mature pollen grain and maintained in a translationally silent state until the progamic phase (Hafidh et al. 2018; Honys et al. 2000; Honys and Twell 2004; Scarpin et al. 2017). Nonetheless, how male gene expression is post-transcriptionally regulated to permit proper pollen guidance is largely unknown and no mRNA regulating factor required for proper pollen ovular guidance has been identified thus far.

In the present article we explore further the role of LARP6C and report that it is a pollen specific factor required for male fertility at the pollen tube guidance step. Our experimental results suggest that LARP6C orchestrates through direct binding the balance between translation, storage and degradation of transcripts that are required for pollen tube guidance. We propose that in dry pollen LARP6C could contribute to maintain its mRNA targets in a translationally silent state and permit their storage in mRNP granules. During pollen tube growth, LARP6C could act to stimulate the translation of at least some of its targets at the site of ovular cue perception

\section{RESULTS}

\section{LARP6C is required for male fertility at the pollen tube guidance step}

Western blotting and high-throughput transcriptomic analyses ((Klepikova et al. 2015; Schmid et al. 2005)) showed that, unlike LARP6A and 6B, LARP6C is almost exclusively expressed in pollen grains and pollen tubes (Figures 1A and S1). We therefore investigated its role in male fertility using two T-DNA insertion mutants, larp6c-3 and larp6c-4. Western blotting showed that both alleles are loss-of-function (lof) mutants (Figure 1B). To establish whether larp6c is a gametophytic mutation, we monitored the transmission efficiency (TE) through the female $\left(T^{f}\right)$ and the male $\left(T^{m}\right)$ gametophytes by reciprocal crosses with wild type and heterozygous larp6c-3/+ or larp6c-4/+ mutants (Figure 1C). We found that both alleles were transmitted normally through the female gametophyte (TEf $6 c-3=100 \%$ and $6 c-4=96.7 \%$ ), whereas their transmission through the male was significantly reduced with $6 c-3$ showing $\mathrm{TE}^{\mathrm{m}}=$ $74.1 \%$ for $6 c-3$ and $6 c-4$ with $\mathrm{TE}^{\mathrm{m}}=68.2 \%$ (Figure $1 \mathrm{C}$ ). This suggests that male but not female fertility is compromised by the loss of LARP6C function.

There are different stages at which the male gametophyte could be deficient, including pollen development, germination, pollen tube growth or guidance and sperm delivery 
(Johnson and Preuss 2002). We monitored these pollen stages in larp6c-3, 6c-4 and wild-type plants (Figures 1D, E and S2A-D). Assessment of male gametophyte maturation by DAPI staining revealed that $10 \%$ of the $6 c-3$ and $6 c-4$ mutant pollen grains did not complete their maturation and were arrested at the bicellular stage, compared to less than $3 \%$ in wild type (Figure S2A).

We next monitored the pollen germination and pollen tube growth using semi in vivo (SIV) ((Palanivelu et al. 2006) and experimental procedure) (Figure S2B, C) and in vitro assays (Figure S2D). We found that both larp6c-3 and $6 c-4$ pollens behave as wild type for these two criteria, demonstrating that LARP6C is not required for pollen germination or pollen tube growth.

We next investigated LARP6C requirement for pollen tube guidance and ovule targeting competence (Johnson and Preuss 2002). We set up SIV experiments using pistils from ms1 plants that are male-sterile mutant with normal function pistil (Van der Veen and Wirtz 1968) to assess the ability of larp6c-deficient pollen tubes to guide through the pistil tissues towards wild-type ovules for fertilization (Figure 1D). Whereas wild-type pollens target $80 \%$ to $100 \%$ of the ovules (with a median value of around $90 \%$ ), both larp $6 c-3$ and $6 c-4$ pollens showed a significant reduction, targeting 50 to $70 \%$ of the wild type ovules (with a median value of $60 \%$ ) (Figure 1D). This failed pollen tube guidance phenotype was complemented by the $\mathrm{N}$ - and $\mathrm{C}$-terminal LARP6C fusion constructs (YFP-LARP6C and LARP6C-tRFP) (Figure 1D and Figure S3A-B). In the competition assays irrespective of the order of pollination or the side of the stigma in which the pollen grains were dusted on, the larp6c-3 and $6 c-4$ pollens also showed a clear deficiency in ovule targeting with a maximum targeting efficiency of $20 \%$ from the expected $50 \%$ rate (Figure S2E). This further supports that the observed guidance phenotype directly relates to larp6c deficiency. We further monitored the ability of the larp6c and larp6c;LARP6C-tRFP complemented pollen to target ovules in planta (Figure 1E). We hand pollinated $m s 1$ pistils and following aniline blue staining, scored the number of targeted and non-targeted ovules. Here again the larp6c deficient pollen tubes displayed only $60 \%$ ovule targeting efficiency versus the $80 \%$ average observed in wild-type pollinated pistils. The targeting efficiency by the larp6c;LARP6C-tRFP complemented lines recovered to a wild-type frequency (Figure 1E).

To verify that larp6c lof specifically affects male fertility, we examined the capability of larp $6 c-3$ and $6 c-4$ mutant ovules to attract wild-type pollen tubes by semi in vivo and in vivo assays and found no significant difference from wild type (Figure S4). In 
summary, our results support that LARP6C is required for male fertility at least in part through its function during pollen tube guidance.

\section{LARP6C forms dynamic cytoplasmic granules in mature pollen grains}

To get a better understanding of LARP6C cellular roles, we firstly monitored its subcellular distribution across pollen maturation and during pollen tube growth (Figure 2 and S3D). In microspores (UNM), LARP6C is only detected in the nucleolus. At the bicellular stage (BCP) LARP6C is much more generally distributed, as it can be detected in the cytoplasm of the vegetative cell (VC) as well as in the vegetative and generative cell nuclei (VCN and GCN). At the tricellular stage (TCP) LARP6C subcellular distribution is similar to that at the bicellular stage, with possibly reduced amounts of LARP6C in the sperm cell nucleus (SCN) compared to other subcellular compartments. In mature pollen grain (MPG) LARP6C displays a complex localization pattern. It is clearly present in the VC cytoplasm, but transitioned from a diffuse pattern to a granular, aggregate-like pattern. In addition to this granular distribution, LARP6C appears to concentrate around the membrane surrounding the vegetative cell nucleus, the sperm cells (SCs) and the cytoplasmic connection (CC) linking the SC1 to the VCN. In pollen tubes, LARP6C no longer showed the granular pattern and is clearly excluded from the SCs.

To determine more precisely the distribution of LARP6C in mature pollen grains, colocalization experiments between LARP6C-tRFP and various markers were undertaken (Figure 3). The histone H2B-GFP fusion protein, expressed under the control of the tomato pollen-specific LAT52 promoter (Twell et al. 1989), was used to label the VCN, thereby demonstrating the absence of LARP6C in this compartment. GEX2 is a sperm cell-expressed transmembrane protein (Mori et al. 2014) that labels the SC membranes and the cytoplasmic connection between SC1 and the VCN (Brownfield et al. 2009; McCue et al. 2011; Mori et al. 2014). Confocal analyses showed that LARP6C and GEX2 display distinct distributions. The tRFP signal is surrounding the GFP-GEX2 signal decorating the membrane of the male germ unit but not the membrane connecting the two sperm cells, supporting that LARP6C is excluded from the sperm cells and therefore only resides in the VC cytoplasm in mature pollen.

We next inquired on the nature of the LARP6C cytoplasmic foci (Figure 3). Eukaryotic cells contain many types of membrane-less cytoplasmic aggregates formed of 
translationally silent mRNAs and RNA binding proteins (RBPs) (Buchan 2014). So far only two types of mRNP granules have been identified in plant cells: processing bodies (p-bodies) and stress granules (SGs) (Weber et al. 2008). Plant p-bodies are - as in other eukaryotes - characterized by the presence of actors of the general mRNA turnover process, such as the DCP1/DCP2 decapping holoenzyme, the XRN4 exoribonuclease or the deadenylation complex. Stress granules on the contrary contain factors belonging to the translational machinery, amongst which the poly $(A)$ binding protein (Weber et al. 2008). First clues on the nature of LARP6C aggregates observed in mature pollen were derived by colocalization experiments with specific markers of p-bodies and SGs respectively, namely DCP1 and PABP. We used a YFPtagged DCP1 protein expressed from its own upstream genomic sequences (Merret et al. 2013a). Although YFP-DCP1 displays a punctuate distribution in the VC cytoplasm, similarly to LARP6C, no obvious colocalization of DCP1 and LARP6C was observed. Of note, DCP1 accumulation appears to be restricted to the VC cytoplasm, at least in mature pollen and contrary to LARP6C, it does not localize at the periphery of the sperm cells or the vegetative nucleus. The Arabidopsis genome contains seven genes (PAB2 to 8) coding for canonical PABPs, including PAB3, 5, 6 and 7 whose transcripts accumulation is restricted to pollen (Honys and Twell 2004; Belostotsky and Meagher 1996; Belostotsky 2003; Chekanova et al. 2001; Chekanova and Belostotsky 2003). We fused the genomic sequence of the PAB5 gene (the most expressed of all four pollen specific $P A B P$ genes) to a YFP tag under the control of its own upstream sequences. In the VC cytoplasm, the YFP-PAB5 protein, displayed a pattern identical to that of the LARP6C protein; it accumulated in foci, surrounding the sperm cells and was excluded from the vegetative nucleus, but, contrary to LARP6C, it is also present in the cytoplasm of the sperm cells.

In summary, our data indicate that LARP6C shows a dynamic subcellular distribution across pollen maturation. We found that in mature pollen LARP6C is restricted to the vegetative cell cytoplasm and is present in aggregates that contain at least one actor of the translation process.

\section{RIP-seq pulldown revealed LARP6C mRNA targets in mature pollen grain}

To explore LARP6C molecular functions we sought to identify its mRNA targets using an unbiased RNA immunoprecipitation and sequencing (RIP-seq) strategy. We conducted the RNA immunoprecipitation procedure from complemented larp $6 c-3$ lof 
mature pollen grains expressing a proLARP6C-FlagHA tagged version (6C-FH here on) (Figure S3A,C) and from wild-type pollen grains as negative control. After assessing the efficiency of the immunoprecipitation through western blotting (Figure S5A), RNAs were extracted from the input and eluate fractions and subjected to RNAseq. After mapping and filtering of the dataset (see supplementary data procedure) a rpkm (read per kilobase per million mapped reads) value was calculated for each protein coding gene. The replicates were found to be reproducible (Figure S5B), enabling us to build table with a mean normalized value per gene and filtered out those that do have at least $1 \mathrm{rpkm}$ value in one of the four samples (input or eluate from wild type or 6C-FH) (Table S1). With such criteria we retained a list of 7356 expressed genes that is expected for a pollen transcriptome which is less complex than that of sporophytic tissues (Loraine et al. 2013).

To find putative LARP6C mRNA targets, we first filtered out transcripts with InputWT/Input-6C-FH ratio $>1.2$ in order to select for transcripts whose levels did not vary between control and 6C-FH inputs. Then we picked transcripts with an RE (RE is the eluate over input ratio) value in the $6 \mathrm{C}-\mathrm{FH}$ samples above 1 , to select for mRNAs that were enriched by the immunoprecipitation procedure (see Figure S5C for a diagram of the filtering workflow). To further increase stringency and reveal mRNAs that are most likely to be direct targets of LARP6C, we compared the RE $\mathrm{E}_{W T}$ to the $\mathrm{RE}_{6 \mathrm{C}-\mathrm{FH}}$ and, based on the repartition of the values (Figure S5D), we selected transcripts with an (RE 6 $\mathrm{FH}_{\mathrm{H}} / \mathrm{RE}_{\mathrm{WT}}$ ) value of 3 or more. Through above series of filters, we identified 115 genes as direct or indirect mRNA targets of LARP6C (Table S1).

No gene ontology (GO) term was found to be significantly enriched amongst the 115 mRNAs identified as potential LARP6C targets. Nonetheless a gene to gene analysis of the proteins they encode shows that $25 \%$ code for factors that are membrane associated, $25 \%$ relate to sensing and signalling and $25 \%$ to membrane synthesis (Table S1). In addition several genes are known to be involved in polarized tip growth. The MPK6 kinase (Guan et al. 2014) and the TFIIB (Zhou et al. 2013) general transcription factor are necessary for pollen tube guidance. Exo70H3 is a subunit of the exocyst secretory complex essential for plasma membrane nanodomains specification that define sites for pollen tube directional growth (Li et al. 2010; Synek et al. 2006; Vukasinovic and Zarsky 2016). WAV2 participates in the regulation of Arabidopsis root directional growth in response to environmental cues (Mochizuki et al. 2005) and the AT3G50910 protein is a homolog of human DCC (Delected in 
Colorectal Cancer), a transmembrane receptor of Netrin, an evolutionarily conserved chemical cue of developing axon guidance. Several other LARP6C RIP targets could be required in pollen tube guidance and growth by acting at the signal perception/transduction levels, including PIRL5 (Forsthoefel et al. 2005), CIPK1 and 14 (Mao et al. 2016) and AT3G54800 which bears a PH and a START lipid binding domains and could therefore be involved in lipid signalling (Tang et al. 2005). Finally, the list of LARP6C targets also includes mRNAs coding for factors involved in cell wall synthesis and organization. This comprises actors of sphingolipid metabolism affecting pollen tube guidance (Tartaglio et al. 2017), such as the DES1-like sphingolipid $\Delta 4$ desaturase (Michaelson et al. 2009) and LCB2 which is involved in the first step of sphingolipid biosynthesis (Teng et al. 2008). Players in tri- or diacylglycerol metabolism, the homeostasis of which is necessary for correct pollen tube growth (Botte et al. 2011; Pleskot et al. 2012) are also present; including TAG1 that catalyzes the first step of the triacylglycerol synthesis pathway, SDP1, a triacylglycerol lipase and MGD2 that catalyses the formation of monogalactosyldiacylglycerol (MGDG) from diacylglycerol (DGDG) (Botte et al. 2011). Strikingly, $66 \%$ of the RIP-identified LARP6C targets are enriched or specifically expressed in pollen as compared to their expression in seedlings, while only $33 \%$ of the whole pollen transcriptome shows such higher or specific expression (Loraine et al. 2013).

\section{LARP6C directly binds to the 5'-UTRs of its mRNA targets}

RNA binding proteins often bind to primary sequence motifs shared between their mRNA targets to regulate their fate. Considering that cis regulatory elements are mostly located in the transcripts 5' and/or 3' untranslated regions (UTRs), we looked for primary sequence motif(s) shared between the UTRs of the putative LARP6C targets. Using the Araport database, we found and retrieved the 5'- and 3'-UTRs for 112 genes and ran a discriminative motif search using a MEME search (at the MEME suite portal (https://meme-suite.org/). Whereas no significant motif could be detected in the 3'-UTRs of LARP6C mRNA targets, we identified two regions we named A-box

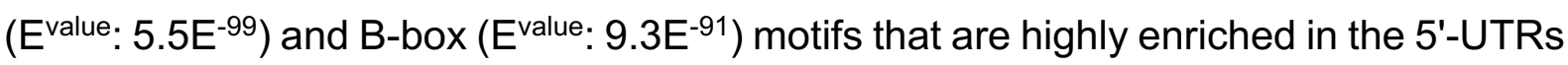
(Figure 4A). As a control we ran an identical motif search using a set of 112 randomly chosen 5'- and 3'-UTRs from genes expressed in pollen according to our transcriptomic analyses. We found no significant enrichment for an A- or a B-type region, supporting that these sequence motifs are specific for mRNAs that are in a complex with LARP6C. 
The A motif is a purine-rich 21 nt sequence, with a clear dominance of A over $G$ nucleotides while the $B$ motif is a pyrimidine-rich 21 nt sequence with a majority of $U$ ( $T$ in DNA) over $C$ repeats (Figure 4A, Table S1). A closer examination of sequences that contain the $A$ and/or $B$ consensus revealed two major categories for each box type. In particular, sequences harboring the A motif are mainly composed of either $A$ or AG/AAG repeats whilst, sequences sharing the $B$ consensus motif contain either $U$ or UC/UUC repeats (Table S1). Amongst the 112 identified 5'-UTRs, 70 (62.5\%) bear the A motif, $74(66 \%)$ the B motif and 49 (43.7\%) carry both (Figure S5E).

The presence of motifs shared by transcripts co-immunoprecipitated with LARP6C in vivo suggests that one or both of these boxes may mediate direct binding of LARP6C. To challenge this hypothesis we tested the RNA binding unit of LARP6C, the Lamodule, for its ability to interact with these boxes in vitro (Figures 4B-E and S6). We designed two oligonucleotide sequences for the $A$ motifs, called $A 1$ and $A 2$ representing the "A-rich" and the "AG/AAG-rich" types respectively (Figure S6A). Analogously B1 and B2 were designed for the B motifs exemplifying the "U-rich" and "UC/UUC-rich" types respectively (Figure 4B). As a control, we designed oligos B3 and $\mathrm{B} 4$ representing respectively $\mathrm{C}$ - or $\mathrm{CU} / \mathrm{CCU}$-rich sequences. A recombinant version of the LARP6C La-module, identical to the one used in Merret et al. (Merret et al. 2013b), was expressed as previously described in E. coli and its ability to bind the A and $B$ type oligo RNAs was quantified by isothermal titration calorimetry (ITC) and electrophoretic mobility shift assay (EMSA) (Figure 4B-E and S6). In the ITC experiments serial titration of LARP6C La-module with RNA oligos B1 and B2 generated in both cases a profile that could be fitted to a sigmoid-shaped binding curve centered around a 1:1 stoichiometry, with a dissociation constant in the low micromolar range (Kds of $2.8 \mu \mathrm{M}$ and 3.7 $\mu \mathrm{M}$ respectively), suggesting a high affinity of LARP6C La-module to B1 and B2 type motifs (Figure 4B and Table S2). The binding mode of LARP6C La-module for the B1 and B2 oligos is similar (but with higher affinity) to that we previously observed for an U20 homopolymer (Table S2 and (Merret et al. 2013b)). On the contrary, oligos B3, B4, A1 and A2 revealed no binding to the recombinant LARP6C domain by ITC in the same experimental conditions used (Figure 4D, Figure S6A and Table S2). Parallel EMSA assays were performed to confirm ITC results, using RNA oligos labelled at the $5^{\prime}$ with $\gamma-{ }^{32} \mathrm{P}$, to monitor RNA-protein complex formation in native gel electrophoresis. Experiments were conducted in the presence or absence of tRNA competitor. For 
oligos showing the ability to form a complex with the La-module, the fraction of bound RNA was plotted against the protein concentration and the dissociation constant determined (Figure 4C). In agreement with ITC, EMSA experiments confirm that B1 and B2 oligos associate with LARP6C La-module, with estimated $K_{d} S$ in the low micromolar range (Kds of $0.8 \mu \mathrm{M}$ and $1.9 \mu \mathrm{M}$ respectively) suggesting here again a high affinity of LARP6C La-module towards B1 and B2 oligos (Figure 4C). B2 binding behaviour is very similar to what we observed for an oligo $\left(\mathrm{U}_{20}\right)$ whereas $B 1$ shows a slightly higher binding affinity. Assays with oligos A1, A2, B3, oligo $\left(\mathrm{C}_{20}\right)$ and to a lesser extent with B4 were hindered by samples being retained in the wells of the gel at high protein concentration (Figure 4E and S6B), an issue most probably linked to protein aggregation (Hellman and Fried 2007). Despite changing various experimental conditions, this issue could not be overcome. The presence of a tRNA competitor marginally helped in the case of B4 albeit association with LARP6C was observable only at high protein concentration and a $\mathrm{Kd}$ could not be calculated as the binding curve did not reach a plateau (Figure 4E).

Collectively these experiments demonstrate that LARP6C La-module binds to oligos B1 and B2 with high affinity and with a similar association mechanism observed with oligo $\left(U_{20}\right)$. B1, which contains three stretches of $U$ residues, one of which 6 uridinelong, appears to bind slightly tighter than $\mathrm{B} 2$ and $\mathrm{U}_{20}$. Altogether these results indicate that the LARP6C La-module strongly binds to B type motifs and does not associate with A type motifs, at least in vitro, and that it displays low affinity for B4.

We hence propose that in vivo LARP6C directly binds to mRNAs that carry in their 5'UTRs, a B1 or B2-type box, to impose posttranscriptional regulation on its mRNA targets.

\section{LARP6C is not required for accumulation of its mRNA targets in mature pollen} grain

To have a global view of LARP6C mode of regulation on its mRNA targets' accumulation we monitored the polyadenylated transcriptome of mature pollen grain in the absence of LARP6C. Pollen grains were collected from wild-type and larp6c-3 plants grown together in a greenhouse (see supplemental experimental procedure), total RNAs extracted and the polyadenylated fraction subjected to next generation sequencing. Following mapping and filtering of the reads (see supplementary experimental procedure), we retained a list of 7399 transcripts with at least 1 rpkm as 
reliably expressed from both Col-0 and larp6c-3 (Figure S7, Table S3). We then used a DE-seq pipeline to find genes whose transcripts differentially accumulate in the mutant background and retained those with a fold change of at least 1.5 between wild type and larp6c-3. We found 2174 genes either up- or downregulated in larp6c-3 pollen (Figure 5A). Monitoring the fold changes of these transcripts between wild type and larp6c-3;6C-FH in the input fraction of the RIP-seq samples, we observed that they are centered around 1 (Figure 5B). This suggests that the expression of the LARP6CFlagHA transgene in the larp6c-3 background fully complemented the larp6c-3 mutation and restores a wild-type level expression, proving that the LARP6C-FlagHA translational fusion is functional and that the misregulation of larp6c-3 pollen transcriptome is biologically significant and the consequence of the loss of LARP6C function. We then asked if the steady-state levels of LARP6C targets are affected in larp6c-3 pollen grains. Of the 115 targets, 96 were detected in the RNA-seq data and only 15 of these differentially accumulated in the larp6c-3 background (Figure 5C, Table S3). Of these ten are downregulated by 1.5 to 2.8 fold and five are upregulated by 1.5 to 1.7 fold in larp6c-3 (Figure 5D). These observations suggest that, at least in dry pollen, LARP6C is not required for maintaining steady-state levels of its mRNA targets.

\section{LARP6C downregulates the translation of a reporter transcript containing a functional 5'-UTR B-box motif}

To gain a mechanistic insight on the impact of LARP6C binding to its mRNA targets, we monitored the expression of a YFP reporter placed under the control of a modified 5'-UTR region belonging to a LARP6C target (At5G20410, MGD2) which carries a potential bona fide B-box and no A-box motif (Table S1). We respectively replaced the native B-box by two sequences for which we proved in vitro that they are strongly bound (B1-box) or not bound at all (B3-box) by LARP6C La-module (Figure 4B-E). We inserted the modified 5'-UTR sequences downstream of the CaMV35S promoter and fused to coding sequence of the YFP (B1-YFP and B3-YFP) on a plant binary vector (Figure 6A). These YFP containing plasmids were transiently expressed in Nicotiana benthamiana leaves individually or co-infiltrated with a plant binary vector expressing a tRFP-tagged LARP6C also under the control of the CaMV35S promoter. Three days post infiltration, leaves were collected and YFP mRNA and protein levels were assessed (Figure 6B and C). Quantitative analyses showed that when the LARP6C 
protein is present, the levels of the B1-YFP transcript are increased by two fold (Figure 6B). Conversely co-expression of LARP6C with the B3-YFP construct did not impact significantly the B3-YFP mRNA levels (Figure 6B). Remarkably, the presence of LARP6C negatively impacted the translation of the B1-YFP transcript significantly reducing YFP protein accumulation (Figure 6C). No altered B3-YFP protein levels was observed in the presence of LARP6C (Figure 6C). We also monitored the subcellular distribution of the tRFP-LARP6C fusion following agroinfiltration. Similar as observed in mature pollen grains (Figures 2, 3 and S3D) and previously in onion epidermis cells following stress exposure (Merret et al. 2013b), LARP6C formed foci in the cytoplasm of Nicotiana leaves (Figure 6D). Since in pollen grains and onion epidermis cells LARP6C foci contain the poly(A) binding protein, we reason that LARP6C likely aggregates into mRNP stress granules also in tobacco. Furthermore, the presence of LARP6C in these foci coupled with the B-Box dependent impact of LARP6C on YFP mRNA levels (Figure 6B) suggest that the higher levels observed for the B1 version results from a post-transcriptional stabilization rather than a transcriptional effect. Hence LARP6C binding downregulates its targets' translation and protect them from decay. This mRNA protective effect of LARP6C is in contrast with our RNA-seq analyses from MPG but might be the result of distinct cellular contexts, i.e sporophytic versus gametophytic (see discussion).

\section{Partial inactivation of monogalactosyldiacyglycerol (MGDG) synthases aggravates the larp6c pollen tube guidance defect.}

Amongst LARP6C mRNA targets several, enriched in pollen, code for proteins required for lipid synthesis including MGD2, the pollen specific (our data and (Wang et al. 2008)) MGDG synthase (Botte et al., 2011). In situ labelling of Arabidopsis pollen tube membranes shows the presence of DGDG. And the chemical inhibition of MGD enzymatic pathway with galvestine-1 causes reduction of MGDG content and downregulates pollen tube elongation rates (Botte et al. 2011), supporting a role for MGD in male fecundation.

MGD2, is likely to be a direct target of LARP6C as it carries in its 5-UTR, a B-box motif with a majority of $U$ over $C$ residues and two stretches of three Us. We reasoned that if one of the molecular bases of larp6c guidance defect relates to the misregulation of MGD2 protein expression or translation, the specific inhibition of its enzymatic activity with galvestine-1 should alleviate or aggravate the larp6c lof phenotype. We hence 
monitored pollen tube elongation rate and pollen tube guidance in the presence of galvestine-1 by SIV assays (Figure 7). As previously reported (Botte et al. 2011), we observed that galvestine-1 application equally reduced pollen tube elongation of wildtype and larp6c-3 mutant pollen (Figure 7A). However, when we monitored pollen tube guidance efficiency, wild-type pollen tubes were only slightly or not affected at all, whereas pollen tubes of larp6c mutants showed considerable decrease in ovule targeting in the presence of galvestine-1 (Figure 7B). In these conditions, only 30 to $60 \%$ of ovules were targeted by larp $6 c$-pollen tubes whereas the mock treated samples showed 60 to $70 \%$ targeting. In some pistil explants, larp6c pollen tubes showed no ovule targeting at all in the presence of MGD inhibitor, a situation never observed with larp6c-3 pollen tubes in the absence of the MGD inhibitor (Figure 1D, 7B). Collectively, these results support a synergic effect between the loss of LARP6C and the pharmacological inhibition of MGD2 synthase activity on pollen tube guidance but not on pollen tube elongation.

\section{DISCUSSION}

We report here that the RNA binding protein LARP6C is essential for male fertility during the fertilization process at the pollen tube guidance step. Considering that SIV and in vivo assays showed an identical penetrance of larp6c pollen tube guidance defect and since the transmitting tract is absent in semi in vivo approaches, we conclude that LARP6C is likely to be involved in the micropylar guidance step. In other words, LARP6C is likely required for pollen tubes to sense and/or respond to ovule emitted cues.

In our study, we identified 115 mRNAs that in mature pollen, are in a complex with LARP6C. RNA-protein binding assays demonstrated that the La-module of LARP6C is able to interact with the B-type motifs that carry a majority of $U$ over $C$ residues and/or stretches of three consecutive Us. This is consistent with our previous observation that LARP6C shows a strong preference for oligo(U) stretches and little or no binding to any other type of homopolymers (Merret et al. 2013b). We hence hypothesize that in vivo LARP6C, through its La-module, interacts directly with transcripts harbouring B-boxes of the B1 or B2 type in their 5'-UTRs (i.e 52 of the 115 transcripts identified by RIP-seq (Table S1)). At this stage we cannot exclude the possibility that LARP6C also directly binds to its other RIP targets through A-boxes, 
perhaps using yet unidentified RNA binding regions outside the La-module. Or that it is in a complex with these other RIP-identified mRNAs but not through direct binding. We propose that the post-transcriptional regulations mediated by LARP6C on the RIPidentified mRNAs in mature pollen are necessary for ovular guidance of the pollen tube. Indeed a gene to gene analysis of the list of LARP6C targets allowed us to recognize genes which functions were directly found to be necessary for pollen tube guidance, polarized tip growth, signalling or membrane synthesis. Considering the role of LARP6C in male fecundation, we anticipate that amongst its target genes several are yet unrecognized actors of the guided growth of the pollen tube. As an example, our work provides evidence that MGD2 is a novel actor of this cellular process.

But then, what is the mechanistic impact of LARP6C binding to the 5'-UTR of its target transcripts? In eukaryotic cells, mRNAs bound by various proteins tend to aggregate in the cytoplasm into self-assembling membraneless structures to form so called mRNP granules. Several types of mRNP granules, defined upon their cellular context, presumed functions and the presence of particular protein markers have been identified and classified (Buchan 2014). But one of their common features is that they all contain repressed mRNAs that are capable of (re)-entering translation following appropriate signals. The use of a transient reporter system in tobacco leaf cells allowed us to observe that the presence of LARP6C upregulates the mRNA levels, likely through increase in mRNA stability, of its targets while antagonizing their translation. In these transient assays LARP6C systematically displayed a punctuate distribution in the cytoplasm that we deem likely to be stress granules. In somatic cells, stress granules are known to protect translationally silent mRNPs that need to be swifty reintroduced in translation after the stress ends (Merret et al. 2017). We propose that the binding of LARP6C at the 5'-UTR acts to repress translation of its mRNA targets and promote their accumulation into aggregates as storage granules and protect them until their translation is reactivated (see model in Figure 8).

We next asked whether LARP6C retains such molecular functions in the non somatic cells i.e MPG and pollen tube. Interestingly, LARP6C forms punctuate foci throughout the vegetative cell cytoplasm and appears to accumulate, also with an aggregate pattern, around the sperm cells and vegetative nucleus. These LARP6C foci fully colocalized with the poly $(A)$ binding protein PAB5 supporting the idea that these aggregates are likely mRNP granules. Scarpin et al. (Scarpin et al. 2017) reported that the SK14 mRNA which was found to be present but translationally silent in Arabidopsis 
and Tobacco MPGs and reactivated at germination (Honys et al. 2000; Scarpin et al. 2017), forms punctuate foci that also contain DCP1 and VARICOSE that are components of p-bodies in somatic cells (Weber et al. 2008). Interestingly, at germination the production of the SK14 protein coincides with the disappearance of SK14 foci, suggesting that mRNAs could be stored until their protein product is required at or along the progamic phase (Scarpin et al. 2017). We hence propose that LARP6C in dry pollen, could help maintaining its targets in aggregates in a translationally silent form until their translation product is required for the pollen tube function.

The stabilization effect of LARP6C binding observed in transient tobacco assays is in contrast with the observation that in pollen grains LARP6C does not seem to control its targets' steady-state levels. In pollen, as in animal gametes, mRNAs ((including LARP6C target transcripts in larp6c lof pollen) were found to be highly stable suggesting that in the male gametophyte the mRNA decay machineries, as translation, are poorly active (Ylstra and McCormick 1999). This assumption is consistent with our observation that DCP1 which is a core constituent of the decapping mRNA turnover complex, localises into foci in mature pollen grains representing quenched DCP1 activities. Indeed, work conducted in animal cells proposes that when components of the mRNA decay machineries are present in p-bodies they are catalytically inactive (Hubstenberger et al. 2017). Therefore, the discrepancies between results from transient assays in tobacco leaf cells and RNA-seq from pollen grains could hence be the consequence of differences in how mRNA decay complex operates between sporophytic versus reproductive cells as exemplified in other cellular machineries such as Transamidase GPI8 GPI-anchoring complex (Liu et al. 2016).

Using a specific chemical inhibitor of MGD2 synthase activity we observed an aggravation of larp6c pollen guidance. This observation suggests that loosing LARP6C-mediated post-transcriptional regulations and downregulating MGDG/DGDG production add up to dampen further pollen tube competence. Hence this suggests that, in pollen tube, LARP6C might stabilize MGD2 mRNAs and/or promote their translation rather than inhibit protein production. But to be consistent with the observation that it is the guidance defect of larp6c pollen tubes that is aggravated and not their growth rate in the presence of galvestine-1, we propose that LARP6C might shift to a translation stimulatory role only when it reaches together with its bound mRNA, the plasma membrane at the site of ovular cue perception to permit the 
synthesis of MGD2 exactly where its synthase activity is needed (see Figure 8). This working model, is consistent with the known role of human LARP6 that shifts from a repressor to an activator role in the coordinated translation of its target mRNAs, according to its associated proteins and subcellular localization ((Zhang and Stefanovic 2016)).

In mammals, the nervous system axons and dendrites need to rapidly extend unidirectionally into polarized cells guided by chemical cues. In this process, reminiscent of the guided growth of the pollen tube, neuronal mRNP transport granules play a crucial role. They act to transport translationally repressed mRNPs to the precise site of their translational activation and protein product function at the tip of an axon (Loya et al. 2010). Our work posits the exciting notion that there could exist a similar system in the process of plant fecundation.

\section{METHODS}

\section{Plant material and growth conditions}

Arabidopsis thaliana ecotype Columbia-0 (Col-0) was used as wild-type reference. The larp6c-3 (SAIL_268E02) (McElver et al. 2001) and larp6c-4 (WiscDsLox293-296invG4) (Woody et al. 2007) lines were respectively ordered from the NASC and ABRC stock centres. Genotyping primers are reported in Table S4. Stable transgenic lines were obtained using the Agrobacterium tumefasciens based floral dip technique (Clough and Bent 1998). For in vitro culture, surface sterilized seeds were sown on synthetic Murashige \& Skoog (MS) medium at $2.20 \mathrm{~g} / \mathrm{L}$ (1/2 MS) with $0.8 \%$ agar and stored for $48 \mathrm{~h}$ at $4^{\circ} \mathrm{C}$ in the dark before being grown under continuous light (50-60 E. $\left.\mathrm{m}^{-2} \cdot \mathrm{s}^{-1}\right)$ at $20^{\circ} \mathrm{C}$. Soil cultures were performed in growth chambers with $16 \mathrm{~h}$ light $\left(100 \quad\right.$ E.m ${ }^{-}$ ${ }^{2} . \mathrm{s}^{-1}$ ) / $8 \mathrm{~h}$ night at $20^{\circ} \mathrm{C}$ with approximately $75 \%$ humidity. See supplemental experimental procedures for growth conditions used to collect mature pollen for RNAseq and RIP-seq experiments.

\section{Cloning and plasmids}

All LARP6C containing fusions (YFP, tagRFP and FLAGHA) were expressed under the upstream genomic sequences (spanning region -1181 to -1 from ATG) of the LARP6C gene (AT3G19090 locus). To obtain the LARP6C-tRFP and LARP6CFLAGHA fusions, we PCR amplified from Col-0 total genomic DNA, the LARP6C 
genomic region starting from nucleotide -1181 from the ATG to the last nucleotide before the stop codon, using primers 543 and 544 (Table S4). The PCR product was inserted at sites $\mathrm{Kpnl}$ and $\mathrm{Nhel}$ either upstream to the tagRFP or FLAG-HA tag into a pCAMBIA1300-based plant binary vector expressing the HPTII (hygromycin) resistance gene, giving rise to vectors $\mathrm{pEB} 16$ and $\mathrm{pEB} 13$. To obtain the YFP-LARP6C expressing binary vector, the genomic region spanning nucleotides -1181 to -1 from the ATG of the LARP6C gene was PCR amplified with primers 974 and 975 from vector pEB13 and cloned at sites Sacl and Kpnl upstream to a gateway entry cassette into a pPZP221 (Hajdukiewicz et al. 1994) based plant binary vector, carrying the AAC1 (gentamycin) resistance gene, giving rise to vector $p 788$. The $L A R P 6 C$ coding genomic region was PCR amplified from total genomic DNA with primers 976-977 and cloned at sites Spel-EcoRV downstream to the YFP tag located between the AttL1 and L2 gateway donor sites, giving rise to plasmid p793. The plant binary vector, p795, was obtained through a gateway cloning reaction between $\mathrm{p} 788$ and 793 . The PAB5 genomic sequence (expanding from -1966 from the ATG to the last nucleotide before the stop codon) was PCR amplified from total genomic DNA with primers eb3 \& eb4 and cloned using the restriction endonucleases $\mathrm{Xbal}$ and $\mathrm{BamHI}$ into vector CTL579 (pCAMBIA-1300) upstream to the GFP tag, giving rise to plasmid pEB3. The binary vector pGEX2-GEX2GFP was obtained from (Mori et al. 2014). Binary plasmids for transient assays were prepared as follow. DNA fragments encompassing a basal CaMV35S promoter without enhancer sequences (Topfer et al. 1987), modified MGD2 5'-UTRs and the YFP CDS were ordered from GeneCust (http://www.genecust.com/fr) and subcloned into a gateway donor vector. DNA casettes were then introduced into a plant binary vector containing the HPTII (hygromycin) resistance gene (vector CTL575, a derivative from pCAMBIA-1300). The LARP6C coding sequence was fused downstream to the tagRFP fluorescent reporter and placed under the control of a strong CaMV35S promoter by gateway recombinaison with the plant binary entry vector p-SITE-6C1 (Martin et al. 2009).

\section{Pollen phenotyping}

Pollen transmission tests were conducted through hand pollination of wild-type pistils with pollen from mutant and complemented homozygous plants. The number of offsprings carrying the mutation was scored either by PCR-based genotyping or by selection of antibiotic/herbicide-resistant seedlings. The number of mutant seedlings 
was expressed as a percentage over the total number of seedlings and transmission efficiency of the mutant gametophyte calculated as TE $=($ (No of mutant $/$ No of WT $) x$ 100). Experiments were conducted in triplicates. Pollen tube guidance efficiency was scored either through semi in vivo (Palanivelu et al., 2006) or in vivo assays by observation of pistils hand pollinated with the mutant pollen of interest and stained with aniline blue (Mori et al., 2006). Emasculated pistils were from $m s 1$ (male sterility 1) heterozygous plants. This garantees that pistils were not contaminated with wild-type pollen as the ms1-1 mutant is male sterile (Van der Veen and Wirtz 1968; Ito et al. 2007). Monitoring of pollen tube guidance in the presence of galvestine-1 was conducted through semi in vivo assays but on plates containing 1\% DMSO for mock treatment or 5 or $10 \mu \mathrm{M}$ of galvestine- 1 with a final concentration of $1 \%$ DMSO.

\section{Confocal microscopy}

Subcellular localization experiments were conducted with an LSM700 confocal microscope (Zeiss) using emission/excitation wavelengths: $405 \mathrm{nM}$ / 420-480 nM for DAPI, 555 nM / 600-700 nM for tRFP and 488 nM / 490-555 nM for YFP and GFP. Mature pollen grains were collected through dipping of open flowers into a DAPI solution (1X PBS pH 7, 0.5\% triton-X100, 1 g/mL 4',6-diamino-2-phenylindole) drop deposited on a glass slide. To collect immature pollen grains from microspore, bi- and tricellular stages, anthers were collected from unopened buds, placed in a DAPI solution drop on a glass slide and gently crushed to free pollen grains from pollen sacs. Pollen tubes germinated and grown as described in the supplemental experimental procedures were directly observed from the germination slide.

\section{RNA related techniques}

Total RNA extractions were performed according to (Logemann et al. 1987). To purify total RNA from mature pollen, 0.5 to $3 \mathrm{mg}$ of dry pollen were grinded with a Silamat S6 (Ivolcar Vivadent, Schaan, Liechtenstein) in $500 \mathrm{~L}$ of GuHCl buffer (8 M GuHCl, 20 mM MES, 20 mM EDTA, at pH 7, 50 mM $\beta$-mercaptoethanol) and with 5 glass beads. Following $1 \mathrm{~min}$ vortexing at full speed and $10 \mathrm{~min}$ incubation on ice, RNAs were separated from proteins with two consecutive phenol-chloroform-IsoAmylAlcool (IAA) $(25: 24: 1)$ extractions, followed by one extraction with chloroform-IAA (24:1), consisting in 1 min vortexing at full speed and 10 min centrifugation at $16^{\circ} \mathrm{C}, 14.000 \mathrm{~g}$. RNAs are then precipitated in the presence of $0.7 \mathrm{vol}$ isopropanol, $1 / 20^{\text {th }} \mathrm{CH}_{3} \mathrm{COOH}$ at $1 \mathrm{M}$ for 
$20 \mathrm{~min}$ at $-20^{\circ} \mathrm{C}$. Following 20 min centrifugation at $4^{\circ} \mathrm{C}, 14.000 \mathrm{~g}$ the RNA pellet is washed in 90\% EtOH and resuspended in RNAse free water. The average efficiency of pollen RNA extraction was at 5-6 $\mu \mathrm{g}$ of RNA per mg of dry pollen.

RNAs were treated with DNAse using the Turbo ${ }^{T M}$ DNAse kit from Ambion (Thermofisher), following the supplier instructions for the "Rigourous DNAse treatment" procedure. For Reverse transcription, $500 \mathrm{ng}$ of DNAse treated RNAs were reverse transcribed with the SuperScript III reverse transcriptase (Life Technologies, Thermo Scientific, USA) and $500 \mathrm{ng}$ of oligodT 18 . PCR reactions were conducted with $1.5 \mu \mathrm{L}\left(1 / 20^{\text {th }}\right.$ of the reverse transcription solution) of cDNA for LARP6C and $2 \mu \mathrm{L}$ for ACTIN8 amplication, using the GoTaq G2 Flexi DNA polymerase from Promega and 30 PCR cycles. For qPCR analyses, total RNA was isolated using the Monarch Total RNA Miniprep Kit (New England Biolabs), and genomic DNA were eliminated by gDNA removal columns and on-column DNasel-treated (New England Biolabs). Following, 5 $\mu \mathrm{g}$ of total RNA were reverse transcribed with the SuperScript IV kit (Life Technology) using an oligod $\mathrm{T}_{18}$ primer. $\mathrm{qPCR}$ reactions were conducted on the LightCycler 480 Multiwell Plates 384-well themocycler (Roche Applied Sciences), with the following amplification program: $5 \min 95^{\circ} \mathrm{C}, 40$ cycles of $15 \mathrm{~s}$ at $95^{\circ} \mathrm{C}$, and $1 \mathrm{~min}$ at $60^{\circ} \mathrm{C}$. After the amplification, a melting curve analysis with a temperature gradient of $0.1^{\circ} \mathrm{C} / \mathrm{s}$ from $60^{\circ} \mathrm{C}$ to $95^{\circ} \mathrm{C}$ was performed. The PCR mix contained Takyon qPCR master mix (Eurogentec), $500 \mathrm{nM}$ gene-specific primers, and $1 \mu \mathrm{L}$ cDNA in a total reaction volume of $10 \mu \mathrm{L}$. Primer efficiencies were determined on standard curves, with 10-fold serial dilution series, ranging from $1 \times 10^{1}$ to $1 \times 10^{6}$ of the cDNA samples. Relative quantification was performed by the $\Delta \Delta \mathrm{C}_{\mathrm{T}}$ method (Livak and Schmittgen 2001), with YFP and HPTII genes used as the target and reference genes, respectively. The sequences of the primers used for RT- and qPCRs are reported in Table S4.

\section{RNA-seq, RIP-seq, bioinformatics}

Techniques related to large scale analyses (RNA-seq and RIP-seq), as well as the bioinformatic analyses are detailed in the supplemental experimental procedure section.

\section{Protein related techniques}

Total protein extracts were separated by SDS PAGE on an acrylamide gel before being electrotransferred on a PVDF membrane $(0.45 \mu \mathrm{M}$, Merck millipore). After saturation 
in a (1X TBS - $0.1 \%$ tween - $5 \%$ fat-free dry milk) solution, membranes were incubated with the appropriate primary then secondary antibodies, washed three times with a $1 \mathrm{X}$ TBS $-0.1 \%$ tween solution, and chemiluminescence revealed with the EMD Millipore immobilon western chemiluminescent HRP substrate kit. Antibodies against the LARP6C protein were custom made (at Agro-Bio, La Ferté St Aubin, France) through immunization of rabbits with peptide "KRTSQFTDRDREELGQ" (amino acids 220235). Anti-LARP6C antibodies are utilized at $1 / 1000^{\text {th }}$ dilution in a (1X TBS $-0.1 \%$ Tween - 5\% fat-free dry milk) solution and incubated overnight at $4{ }^{\circ} \mathrm{C}$ with gentle shaking. Secondary antibodies were anti-rabbit HRP (Bio-Rad) and used at a $1 / 5,000^{\text {th }}$ dilution. Anti-ACTIN antibodies were from Affinity Bio Reagents (Golden, CO, USA) and used at $1 / 15,000^{\text {th }}$ dilution. Secondary antibodies were anti-mouse HRP (Bio-Rad) used at a $1 / 10,000^{\text {th }}$ dilution. For transient assays, the YFP protein was detected using the GFP antibody (Clontech) at $1 / 5,000^{\text {th }}$ and the UGPase antibody (Agrisera) at $1 / 7,500^{\text {th }}$ dilution, both incubated overnight at $4^{\circ} \mathrm{C}$. Secondary antibodies anti-mouse HRP (Bio-Rad) used at a $1 / 2,000^{\text {th }}$ dilution, and anti-rabbit HRP (Bio-Rad) used at a $1 / 5,000^{\text {th }}$ dilution, were incubated, respectively for YFP and UGPase, for $1 \mathrm{~h}$ at room temperature .

The LARP6C La-module (amino acids 137-223) was recombinantly expressed as in (Merret et al. 2013b). The hexahistidine tag (His-tag) was removed by proteolysis through incubation at $4^{\circ} \mathrm{C}$ overnight with $\mathrm{HRV} 3 \mathrm{C}$ Protease in $50 \mathrm{mM}$ Tris- $\mathrm{HCl} \mathrm{pH} \mathrm{7.25,}$ $100 \mathrm{mM} \mathrm{KCl}, 0.2$ mM EDTA, 1 mM DTT, 10\% gycerol buffer. The cleaved His-Tag and the protease were separated from the La-module through retention onto a Ni-NTA column. The protein was then subjected to heparin column purification and dialysis in a final buffer as in (Merret et al. 2013b).

\section{ITC and EMSA}

RNA oligos were ordered as custom made from lba-Lifescience GmbH (Göttingen, Germany) at a $1 \mu$ Molar scale and HPLC purified. The lyophilized RNAs were resuspended in RNAse free water (DEPC treated) at concentrations ranging from 2.5 to $3 \mathrm{mM}$. The ITC experiments were performed as in (Merret et al. 2013b) on a iTC200 microcalorimenter (Malvern). Here, 20 injections of $2 \mu \mathrm{L}$ RNA solutions at 200-320 $\mu \mathrm{M}$ concentration were added to protein solutions (6C-La-module) at 20-30 $\mu \mathrm{M}$ with a computer-controlled $40 \mu \mathrm{L}$ syringe. Control titrations of RNA into buffer alone and into 
protein solution without the His-tag were performed (not shown). The heat per injection normalized per mole of injectant versus molecular ratio, was analyzed with the MicroCal-Origin 7.0 software package and fitted using a nonlinear least-square minimization algorithm using a theoretical single-site binding model. $\Delta \mathrm{H}$ (reaction enthalpy change in $\mathrm{kcal} / \mathrm{mol}$ ), $\mathrm{K}_{\mathrm{b}}$ (binding constant equal to $1 / \mathrm{Kd}$ ), and $\mathrm{n}$ (molar ratio between the two proteins in the complex) were the fitting parameters. The reaction entropy was calculated using the relationships $\Delta \mathrm{G}=-\mathrm{RT} \ln \mathrm{K}_{\mathrm{b}}(\mathrm{R}=1.986 \mathrm{cal} / \mathrm{mol} \mathrm{K} ; \mathrm{T}$ $=298 \mathrm{~K}$ ) and $\Delta \mathrm{G}=\Delta \mathrm{H}-\mathrm{T} \Delta \mathrm{S}$. All ITC experiments were repeated at least three times, and a detailed analysis of the thermodynamic parameters and errors calculated as the standard deviation from the mean value are reported in Table S2. For EMSA (Electrophoretic Mobility Shift Assay), RNAs were 5'-labelled with $\gamma$ - ${ }^{32} \mathrm{P}$-ATP using the T4 polynucleotide kinase. Recombinant protein and RNA were mixed and incubated for 15 minutes at room temperature in $(20 \mathrm{mM}$ Tris $\mathrm{pH} 7.25,200 \mathrm{mM} \mathrm{KCl}, 5 \%$ of glycerol, $1 \mathrm{mM}$ DTT, $0.1 \mathrm{mg} / \mathrm{mL}$ of BSA and 0.7 units of RNAse inhibitors (RNAseOUT, Invitrogen)). Each reaction mixture $(22 \mu \mathrm{L})$ contained $3 \mathrm{nM}$ of labelled RNA oligo and varying amounts of LARP6C La-module (3-fold serial dilutions from a $88 \mu \mathrm{M}$ sample). The experiments were performed in the absence or in the presence of unlabelled tRNAmix of E. coli MRE $600(0.01 \mathrm{mg} / \mathrm{mL})$. After the addition of $2 \mu \mathrm{L}$ of $30 \%$ Ficoll, the samples were loaded on a $9 \%$ native polyacrylamide gel prerun at $100 \mathrm{mV}$ for 1 hour at $4^{\circ} \mathrm{C}$ in $0.5 x$ TBE (Tris-borate-EDTA buffer). A typical EMSA experiment was run at $4^{\circ} \mathrm{C}$ for 1 hour at $125 \mathrm{mV}$. Gels were dried onto 3MM chromatography paper and then exposed to a phosphoimaging plate overnight. These were then analysed on a phosphoimager Typhoon Trio and quantified with Image Quant TL software. The fraction of bound RNA was plotted versus the protein concentrations. To determine the dissociation constants the data were fitted with Origin 8.0 to a modified Hill equation (Ryder et al. 2008) using non-linear least squares methods and assuming a 1:1 stoichiometry.

\section{Nicotiana benthamiana transient assays}

Plasmids B1-YFP, B3-YFP and RFP-LARP6C were respectively transformed into Agrobacterium tumefasciens strain LB4404. Transformed bacteria were cultivated until they reached OD 0.8 and used to infiltrate fully expanded leaves from eight-week-old N.benthamina plants, using needless syringe, as described in (Ruiz et al. 1998). When 
double transformations were performed (B1-YFP and RFP-LARP6C or B3-YFP and RFP-LARP6C) an equimolar mixture of Agrobacterium cultures (OD 0.4) was used for transformation. For each transformation we also used an Agrobacterium culture carrying a plasmid (OD 0.2) allowing the expression of the P19 suppressor of RNA silencing (Voinnet et al. 2003). Three days post-infiltration (3 dpi), transformed leaf segments were collected, flash frozen and total RNA or protein extracted. Alternatively, $3 \mathrm{dpi}$, transformed leaves were observed by confocal microscopy to monitor YFP and RFP signals.

\section{Accession Numbers}

Sequence data in this study can be found under the following accession numbers : Bioproject PRJNA557669. RNA-Seq-Col0-Rep 1: SRR9887494; RNA-Seq-Col0-Rep 1: SRR9887493; RNA-Seq-larp6c-3-Rep 1; SRR9887496; RNA-Seq-larp6c-3-Rep 2 SRR9887495; RIP-Col0-Input-Rep 1: SRR9887498; RIP-Col0-Eluate-Rep 1: SRR9887500; RIP-Col0-Input-Rep 2: SRR9887497; RIP-Col0-Eluate-Rep 2: SRR9887499; RIP-LARP6C-Input-Rep 1: SRR9887490; RIP-LARP6C-Eluate-Rep 1: SRR9887492; RIP-LARP6C-Input-Rep 2: SRR9887489; RIP-LARP6C-Eluate-Rep 2 : SRR9887491.

\section{Supplemental data}

Table S1: RIP-seq experiment: rpkm values for genes detected in the input and eluate fractions of wild type and 6C-FH pollen grain. List of putative LARP6C mRNA targets with a description of the two 5'-UTR enriched motifs.

Table S2: Thermodynamic parameters to the calorimetric analyses shown in Figure 4B, 4D and S6A.

Table S3: RNA-Seq experiment: rpkm values for genes detected in wild type and larp6c-3 mature pollen grain. List of genes differentially expressed between larp6c-3 and wild type.

Table S4: Primers used for genotyping, cloning and RT-PCR. Sequence, efficiency of qPCR primers, amplicon sizes and Tms.

Supplemental Figure 1: Expression profiles of $L A R P 6 A, 6 B$ and $6 C$ mRNAs across development. Supports Figure $1 \mathrm{~A}$. 
Supplemental Figure 2: Pollen maturation, germination and pollen tube growth of larp6c lof mutants. Supports Figure 1D-E.

Supplemental Figure 3: Schematic representation of LARP6C transgenes and western blotting analyses of their accumulation. Subcellular distribution of LARP6C tagged with tagRFP at its C-terminus in bicellular, tricellular and mature pollen grain. Supports Figures 1D-E, 2 and 3.

Supplemental Figure 4: Pollen attraction competence of larp6c-3 and $6 c 4$ ovules. Supports Figure 1C-E.

Supplemental Figure 5: Reproducibility of the RIP-seq data, RIP-seq filtering workflow, representation of the position of A and/or B boxes on the 5'-UTRs of LARP6C targets. Supports Figure 4A-B.

Supplemental Figure 6: ITC and EMSA assessment of LARP6C La-module binding to A type oligos. Supports Figure 4C-F.

Supplemental Figure 7: Reproducibility of the RNA-seq data. Supports Figure 5.

Supplemental text: Supplemental figure legends and supplemental methods.

\section{Funding}

This work was funded by the CNRS, The University of Perpignan (UPVD), the Agence Nationale pour la Recherche (ANR) grant Heat-EpiRNA ( $n^{\circ}$ : ANR-17-CE20-007-01); a Bonus Quality Research (BQR) funded by the University of Perpignan ; a Collaborative PICS Project (LARP\&STRESS, $n^{\circ}$ 6170) funded by CNRS; Grantová agentura České republiky (GACR) grants ( $\mathrm{n}^{\circ}: 17-23203 \mathrm{~S}$ and 18-02448S), European Regional Development Fund-Project "Centre for Experimental Plant Biology" (No. CZ.02.1.01/0.0/0.0/16_019/0000738) and the Royal Society Newton International fellowship (ref. NF140482).

\section{Acknowledgements:}

This work was supported by the CNRS, the University of Perpignan (UPVD), the Institut Universitaire de France (IUF) and the Bio-Environnement platform through utilization of the confocal microscope. This study is set within the framework of the "Laboratoires d'Excellence (LABEX)" TULIP (ANR-10-LABX-41). EB was the recipient of a PhD grant from the UPVD, Doctoral School ED305. CGL jr. is the recipient of a short-term contract supported by the ANR Heat-EpiRNA (ANR-17-CE20-007-01). SH, KK and DH are supported by GACR grant numbers 17-23203S and 18-02448S. ICG was supported 
by a Royal Society Newton International fellowship (ref. NF140482). IGC and MRC thank the Centre for Biomolecular Spectroscopy at King's College London funded by a capital award from the Wellcome Trust. We would like to thank E. Maréchal (LPCV, Grenoble, France) for kindly providing us galvestine-1 and R. Merret (LGDP, Perpignan, France) fo critical reading of the manuscript.

\section{References}

Belostotsky DA (2003) Unexpected complexity of poly(A)-binding protein gene families in flowering plants: three conserved lineages that are at least 200 million years old and possible auto- and cross-regulation. Genetics 163 (1):311-319

Belostotsky DA, Meagher RB (1996) A pollen-, ovule-, and early embryo-specific poly $(A)$ binding protein from Arabidopsis complements essential functions in yeast. The Plant cell 8 (8):1261-1275. doi:10.1105/tpc.8.8.1261

Botte CY, Deligny M, Roccia A, Bonneau AL, Saidani N, Hardre H, Aci S, YamaryoBotte Y, Jouhet J, Dubots E, Loizeau K, Bastien O, Brehelin L, Joyard J, Cintrat JC, Falconet D, Block MA, Rousseau B, Lopez R, Marechal E (2011) Chemical inhibitors of monogalactosyldiacylglycerol synthases in Arabidopsis thaliana. Nature chemical biology 7 (11):834-842. doi:10.1038/nchembio.658

Bousquet-Antonelli C, Deragon J (2009) A comprehensive analysis of the La-motif protein superfamily. RNA $15(5): 750-764$

Brownfield L, Hafidh S, Borg M, Sidorova A, Mori T, Twell D (2009) A plant germlinespecific integrator of sperm specification and cell cycle progression. PLoS Genet 5 (3):e1000430. doi:10.1371/journal.pgen.1000430

Buchan JR (2014) mRNP granules. Assembly, function, and connections with disease. RNA biology 11 (8):1019-1030. doi:10.4161/15476286.2014.972208

Cai L, Fritz D, Stefanovic L, Stefanovic B (2010a) Binding of LARP6 to the conserved 5 ' stem-loop regulates translation of mRNAs encoding type I collagen. J Mol Biol 395 (2):309-326

Cai L, Fritz D, Stefanovic L, Stefanovic B (2010b) Nonmuscle myosin-dependent synthesis of type I collagen. J Mol Biol 401 (4):564-578

Challa AA, Stefanovic B (2011) A novel role of vimentin filaments: binding and stabilization of collagen mRNAs. Molecular and cellular biology 31 (18):37733789. doi:10.1128/MCB.05263-11

Chekanova JA, Belostotsky DA (2003) Evidence that poly(A) binding protein has an evolutionarily conserved function in facilitating mRNA biogenesis and export. RNA 9 (12):1476-1490

Chekanova JA, Shaw RJ, Belostotsky DA (2001) Analysis of an essential requirement for the poly $(A)$ binding protein function using cross-species complementation. Current biology : CB 11 (15):1207-1214

Clough SJ, Bent AF (1998) Floral dip: a simplified method for Agrobacterium-mediated transformation of Arabidopsis thaliana. The Plant journal : for cell and molecular biology $16(6): 735-743$

Feng QN, Liang X, Li S, Zhang Y (2018) The ADAPTOR PROTEIN-3 Complex Mediates Pollen Tube Growth by Coordinating Vacuolar Targeting and Organization. Plant physiology 177 (1):216-225. doi:10.1104/pp.17.01722 
Forsthoefel NR, Cutler K, Port MD, Yamamoto T, Vernon DM (2005) PIRLs: a novel class of plant intracellular leucine-rich repeat proteins. Plant \& cell physiology 46 (6):913-922. doi:10.1093/pcp/pci097

Guan Y, Lu J, Xu J, McClure B, Zhang S (2014) Two Mitogen-Activated Protein Kinases, MPK3 and MPK6, Are Required for Funicular Guidance of Pollen Tubes in Arabidopsis. Plant physiology 165 (2):528-533. doi:10.1104/pp.113.231274

Hafidh S, Potesil D, Fila J, Fecikova J, Capkova V, Zdrahal Z, Honys D (2014) In search of ligands and receptors of the pollen tube: the missing link in pollen tube perception. Biochemical Society transactions 42 (2):388-394. doi:10.1042/BST20130204

Hafidh SS, Potesil D, Muller K, Fila J, Michailidis C, Herrmannova A, Fecikova J, Ischebeck T, Valasek LS, Zdrahal Z, Honys D (2018) Dynamics of Nicotiana tabacum pollen sequestrome defined by subcellular coupled omics. Plant physiology. doi:10.1104/pp.18.00648

Hajdukiewicz P, Svab Z, Maliga P (1994) The small, versatile pPZP family of Agrobacterium binary vectors for plant transformation. Plant molecular biology 25 (6):989-994

Hellman LM, Fried MG (2007) Electrophoretic mobility shift assay (EMSA) for detecting protein-nucleic acid interactions. Nature protocols 2 (8):1849-1861. doi:10.1038/nprot.2007.249

Higashiyama T, Takeuchi $\mathrm{H}$ (2015) The mechanism and key molecules involved in pollen tube guidance. Annu Rev Plant Biol 66:393-413. doi:10.1146/annurevarplant-043014-115635

Honys D, Combe J, Twell D, Capkova V (2000) The translationally repressed pollenspecific ntp303 mRNA is stored in non-polysomal mRNPs during pollen maturation. Plant Sexual Reproduction 13 (3):135-144

Honys D, Twell D (2004) Transcriptome analysis of haploid male gametophyte development in Arabidopsis. Genome biology 5 (11):R85

Hubstenberger A, Courel M, Benard M, Souquere S, Ernoult-Lange M, Chouaib R, Yi Z, Morlot JB, Munier A, Fradet M, Daunesse M, Bertrand E, Pierron G, Mozziconacci J, Kress M, Weil D (2017) P-Body Purification Reveals the Condensation of Repressed mRNA Regulons. Molecular cell 68 (1):144-157 e145. doi:10.1016/j.molcel.2017.09.003

Ito T, Nagata N, Yoshiba Y, Ohme-Takagi M, Ma H, Shinozaki K (2007) Arabidopsis MALE STERILITY1 encodes a PHD-type transcription factor and regulates pollen and tapetum development. The Plant cell 19 (11):3549-3562. doi:10.1105/tpc.107.054536

Johnson MA, Preuss D (2002) Plotting a course: multiple signals guide pollen tubes to their targets. Developmental cell 2 (3):273-281

Klepikova AV, Logacheva MD, Dmitriev SE, Penin AA (2015) RNA-seq analysis of an apical meristem time series reveals a critical point in Arabidopsis thaliana flower initiation. BMC genomics 16:466. doi:10.1186/s12864-015-1688-9

Li S, van Os GM, Ren S, Yu D, Ketelaar T, Emons AM, Liu CM (2010) Expression and functional analyses of EXO70 genes in Arabidopsis implicate their roles in regulating cell type-specific exocytosis. Plant physiology 154 (4):1819-1830. doi:10.1104/pp.110.164178

Liu X, C. C, Wang Y, Noble J, Ponvert N, Bundy M, Hoel C, Shpak E, Palanivelu R (2016) The Role of LORELEI in Pollen Tube Reception at the Interface of the 
Synergid Cell and Pollen Tube Requires the Modified Eight-Cysteine Motif and the Receptor-Like Kinase FERONIA. The Plant cell 28 (5):1035-1052

Livak KJ, Schmittgen TD (2001) Analysis of relative gene expression data using realtime quantitative PCR and the 2(-Delta Delta C(T)) Method. Methods 25 (4):402408. doi:10.1006/meth.2001.1262

Logemann J, Schell J, Willmitzer L (1987) Improved method for the isolation of RNA from plant tissues. Analytical biochemistry 163 (1):16-20

Loraine AE, McCormick S, Estrada A, Patel K, Qin P (2013) RNA-seq of Arabidopsis pollen uncovers novel transcription and alternative splicing. Plant physiology 162 (2):1092-1109. doi:10.1104/pp.112.211441

Loya CM, Van Vactor D, Fulga TA (2010) Understanding neuronal connectivity through the post-transcriptional toolkit. Genes \& development 24 (7):625-635. doi:10.1101/gad.1907710

Manojlovic Z, Earwood R, Kato A, Perez D, Cabrera OA, Didier R, Megraw TL, Stefanovic B, Kato $Y(2017)$ La-related protein 6 controls ciliated cell differentiation. Cilia 6:4. doi:10.1186/s13630-017-0047-7

Mao J, Manik SM, Shi S, Chao J, Jin Y, Wang Q, Liu H (2016) Mechanisms and Physiological Roles of the CBL-CIPK Networking System in Arabidopsis thaliana. Genes (Basel) 7 (9). doi:10.3390/genes7090062

Maraia RJ, Mattijssen S, Cruz-Gallardo I, Conte MR (2017) The La and related RNAbinding proteins (LARPs): structures, functions, and evolving perspectives. Wiley interdisciplinary reviews RNA. doi:10.1002/wrna.1430

Martin K, Kopperud K, Chakrabarty R, Banerjee R, Brooks R, Goodin MM (2009) Transient expression in Nicotiana benthamiana fluorescent marker lines provides enhanced definition of protein localization, movement and interactions in planta. The Plant journal : for cell and molecular biology 59 (1):150-162

Martino L, Pennell S, Kelly G, Busi B, Brown P, Atkinson RA, Salisbury NJ, Ooi ZH, See KW, Smerdon SJ, Alfano C, Bui TT, Conte MR (2015) Synergic interplay of the La motif, RRM1 and the interdomain linker of LARP6 in the recognition of collagen mRNA expands the RNA binding repertoire of the La module. Nucleic acids research 43 (1):645-660. doi:10.1093/nar/gku1287

McCue AD, Cresti M, Feijo JA, Slotkin RK (2011) Cytoplasmic connection of sperm cells to the pollen vegetative cell nucleus: potential roles of the male germ unit revisited. Journal of experimental botany $62 \quad(5): 1621-1631$. doi:10.1093/jxb/err032

McElver J, Tzafrir I, Aux G, Rogers R, Ashby C, Smith K, Thomas C, Schetter A, Zhou Q, Cushman MA, Tossberg J, Nickle T, Levin JZ, Law M, Meinke D, Patton D (2001) Insertional mutagenesis of genes required for seed development in Arabidopsis thaliana. Genetics 159 (4):1751-1763

Merret R, Carpentier MC, Favory JJ, Picart C, Descombin J, Bousquet-Antonelli C, Tillard P, Lejay L, Deragon JM, Charng YY (2017) Heat Shock Protein HSP101 Affects the Release of Ribosomal Protein mRNAs for Recovery after Heat Shock. Plant physiology 174 (2):1216-1225. doi:10.1104/pp.17.00269

Merret R, Descombin J, Juan Y, Favory J, Carpentier M, Chaparro C, Charng Y, Deragon J, Bousquet-Antonelli C (2013a) XRN4 and LARP1 are required for a heat-triggered mRNA decay pathway involved in plant acclimation and survival during thermal stress. Cell reports 5:1279-1293

Merret R, Martino L, Bousquet-Antonelli C, Fneich S, Descombin J, Billey E, Conte MR, Deragon JM (2013b) The association of a La module with the PABPinteracting motif PAM2 is a recurrent evolutionary process that led to the 
neofunctionalization of La-related proteins. RNA 19 (1):36-50. doi:10.1261/rna.035469.112

Michaelson LV, Zauner S, Markham JE, Haslam RP, Desikan R, Mugford S, Albrecht S, Warnecke D, Sperling P, Heinz E, Napier JA (2009) Functional characterization of a higher plant sphingolipid Delta4-desaturase: defining the role of sphingosine and sphingosine-1-phosphate in Arabidopsis. Plant physiology 149 (1):487-498. doi:10.1104/pp.108.129411

Mochizuki S, Harada A, Inada S, Sugimoto-Shirasu K, Stacey N, Wada T, Ishiguro S, Okada K, Sakai T (2005) The Arabidopsis WAVY GROWTH 2 protein modulates root bending in response to environmental stimuli. The Plant cell 17 (2):537-547. doi:10.1105/tpc.104.028530

Mori T, Igawa T, Tamiya G, Miyagishima SY, Berger F (2014) Gamete attachment requires GEX2 for successful fertilization in Arabidopsis. Current biology : CB 24 (2):170-175. doi:10.1016/j.cub.2013.11.030

Palanivelu R, Preuss D, Weterings K, Russell S, Lord E, Russell S, Huck N, Moore J, Federer M, Grossniklaus U (2006) Distinct short-range ovule signals attract or repel Arabidopsis thaliana pollen tubes in vitro. BMC plant biology 6:7. doi:10.1186/1471-2229-6-7

Pleskot R, Pejchar P, Bezvoda R, Lichtscheidl IK, Wolters-Arts M, Marc J, Zarsky V, Potocky M (2012) Turnover of Phosphatidic Acid through Distinct Signaling Pathways Affects Multiple Aspects of Pollen Tube Growth in Tobacco. Frontiers in plant science 3:54. doi:10.3389/fpls.2012.00054

Ruiz MT, Voinnet O, Baulcombe DC (1998) Initiation and maintenance of virus-induced gene silencing. The Plant cell 10 (6):937-946. doi:10.1105/tpc.10.6.937

Ryder SP, Recht MI, Williamson JR (2008) Quantitative analysis of protein-RNA interactions by gel mobility shift. Methods Mol Biol 488:99-115. doi:10.1007/978-1-60327-475-3_7

Scarpin MR, Sigaut L, Temprana SG, Boccaccio GL, Pietrasanta LI, Muschietti JP (2017) Two Arabidopsis late pollen transcripts are detected in cytoplasmic granules. Plant Direct 1 (4):1-10. doi:10.1002/pld3.12

Schmid M, Davison TS, Henz SR, Pape UJ, Demar M, Vingron M, Scholkopf B, Weigel D, Lohmann JU (2005) A gene expression map of Arabidopsis thaliana development. Nat Genet 37 (5):501-506. doi:10.1038/ng1543

Synek L, Schlager N, Elias M, Quentin M, Hauser MT, Zarsky V (2006) AtEXO70A1, a member of a family of putative exocyst subunits specifically expanded in land plants, is important for polar growth and plant development. The Plant journal : for cell and molecular biology 48 (1):54-72. doi:10.1111/j.1365313X.2006.02854.x

Tang D, Ade J, Frye CA, Innes RW (2005) Regulation of plant defense responses in Arabidopsis by EDR2, a PH and START domain-containing protein. The Plant journal : for cell and molecular biology 44 (2):245-257. doi:10.1111/j.1365313X.2005.02523.x

Tartaglio V, Rennie EA, Cahoon R, Wang G, Baidoo E, Mortimer JC, Cahoon EB, Scheller HV (2017) Glycosylation of inositol phosphorylceramide sphingolipids is required for normal growth and reproduction in Arabidopsis. The Plant journal : for cell and molecular biology 89 (2):278-290. doi:10.1111/tpj.13382

Teng C, Dong H, Shi L, Deng Y, Mu J, Zhang J, Yang X, Zuo J (2008) Serine palmitoyltransferase, a key enzyme for de novo synthesis of sphingolipids, is essential for male gametophyte development in Arabidopsis. Plant physiology 146 (3):1322-1332. doi:10.1104/pp.107.113506 
Topfer R, Matzeit V, Gronenborn B, Schell J, Steinbiss HH (1987) A set of plant expression vectors for transcriptional and translational fusions. Nucleic acids research 15 (14):5890. doi:10.1093/nar/15.14.5890

Twell D, Wing R, Yamaguchi J, McCormick S (1989) Isolation and expression of an anther-specific gene from tomato. Molecular \& general genetics : MGG 217 (23):240-245

Van der Veen JH, Wirtz P (1968) EMS-induced genic male sterility in Arabidopsis thaliana: a model selection experiment Euphytica 17:371-377

Voinnet O, Rivas S, Mestre P, Baulcombe D (2003) An enhanced transient expression system in plants based on suppression of gene silencing by the p19 protein of tomato bushy stunt virus. The Plant journal : for cell and molecular biology 33 (5):949-956

Vukasinovic N, Zarsky V (2016) Tethering Complexes in the Arabidopsis Endomembrane System. Front Cell Dev Biol 4:46. doi:10.3389/fcell.2016.00046

Vukmirovic M, Manojlovic Z, Stefanovic B (2013) Serine-threonine kinase receptorassociated protein (STRAP) regulates translation of type I collagen mRNAs. Molecular and cellular biology 33 (19):3893-3906. doi:10.1128/MCB.00195-13

Wang Y, Zhang WZ, Song LF, Zou JJ, Su Z, Wu WH (2008) Transcriptome analyses show changes in gene expression to accompany pollen germination and tube growth in Arabidopsis. Plant physiology 148 (3):1201-1211. doi:10.1104/pp.108.126375

Weber C, Nover L, Fauth M (2008) Plant stress granules and mRNA processing bodies are distinct from heat stress granules. The Plant journal : for cell and molecular biology 56 (4):517-530. doi:10.1111/j.1365-313X.2008.03623.x

Weng H, Kim C, Valavanis C, Wang Z, Schwartz LM (2009) Acheron, an novel LA antigen family member, binds to CASK and forms a complex with Id transcription factors. Cell Mol Biol Lett 14 (2):273-287. doi:10.2478/s11658-0080046-1

Woody ST, Austin-Phillips S, Amasino RM, Krysan PJ (2007) The WiscDsLox T-DNA collection: an arabidopsis community resource generated by using an improved high-throughput T-DNA sequencing pipeline. J Plant Res 120 (1):157-165. doi:10.1007/s10265-006-0048-x

Ylstra B, McCormick S (1999) Analysis of mRNA stabilities during pollen development and in BY2 cells. The Plant journal : for cell and molecular biology 20 (1):101108

Zhang Y, Stefanovic B (2016) LARP6 Meets Collagen mRNA: Specific Regulation of Type I Collagen Expression. Int J Mol Sci 17 (3):419. doi:10.3390/ijms17030419

Zhou JJ, Liang Y, Niu QK, Chen LQ, Zhang XQ, Ye D (2013) The Arabidopsis general transcription factor TFIIB1 (AtTFIIB1) is required for pollen tube growth and endosperm development. Journal of experimental botany 64 (8):2205-2218. doi:10.1093/jxb/ert078

\section{FIGURE LEGENDS}

Figure 1: LARP6C is required for pollen tube guidance. (A), Western blot analysis of steady-state LARP6C protein accumulation in seedling roots (lane 1) and aerial parts (lane 2), cauline leaves (lane 3), stem (lane 4), whole flower (lane 5) and mature 
pollen grain (lane 6). The same blot was probed with antibodies against LARP6C or ACTIN as loading control. (B), larp6c-3 and larp6c-4 alleles are loss-of-function mutants. Top panel : schematic representation of the LARP6C gene and LARP6C protein. Plain boxes represent exons and lines represent introns. The color code for exons is identical to that for the protein conserved domains. Green: PAM2 motif, red: La Motif, blue: RRM1 and yellow: LSA. The insertion sites of the T-DNAs in larp6c-3 and 6c-4 mutants are reported. Bottom panel: western blot analysis of LARP6C accumulation in flowers from wild-type, larp $6 c-3$ and $6 c-4$ lines. ACTIN is used as loading control. (C), Transmission efficiencies of larp6c-3 and $6 c-4$ mutant alleles. Transmission Efficiency (TE) was calculated as: (([No of mutant]/[No of wild type]) $x$ 100). ns: the number of mutant seedlings in the progeny is not significantly different from the expected number of mutant seedlings. (D), Semi in vivo (SIV) pollen tube guidance assays. Wild-type ovules were arranged around homozygous ms1 pistil explants pollinated with pollen from various homozygous backgrounds. The number of targeted (blue boxes) and non-targeted (white boxes) ovules was scored and results are represented as whisker boxplots. Above the graph are a cartoon and a representative pictograph of the setting up of a semi In vivo assay. (E), In vivo pollen tube guidance assays. Homozygous $m s 1$ pistils were pollinated in planta with pollen from various homozygous genotypes, and the number of pollen tube-targeted (blue boxes) and non-targeted ovules (white boxes) were scored following aniline blue staining. Results are reported as whisker boxplots. On (D) and (E) the dotted lines respectively show the median values of targeted (green) and non-targeted (red) ovules by wild-type pollens.

Figure 2: LARP6C shows a dynamic subcellular distribution in pollen. Subcellular localization of fluorescently tagged YFP-LARP6C by confocal microscopy at different stages of pollen maturation: UNM : uninucleate microspore, BCP: bicellular pollen, TCP: tricellular pollen, MPG: mature pollen grain and Pollen tube. Arrows indicate the position of the Nucleolus (No), Generative Cell Nucleus (GCN), Vegetative Nucleus (VCN), Sperm Cell Nucleus (SCN) and Cytoplasmic Connection (CC). Scale bars correspond to $5 \mu \mathrm{M}$. Representative images of LARP6C fused at its C-terminus with a tagRFP reporter are shown in supplemental Figure 2D. 


\section{Figure 3: LARP6C forms mRNP aggregates that contain the $\operatorname{Poly}(A)$ Binding} Protein in the vegetative cell cytoplasm. Confocal observation of mature pollen grains that stably coexpress the LARP6C-tRFP fusions and: the H2B-GFP fusion, the GFP-GEX2 fusion, the YFP-DCP1 fusion or the PAB5-GFP fusion. Except for the H2BGFP marker which is expressed from the tomato LAT52 promoter, all marker genes are expressed from their own upstream genomic sequences. Arrows indicate the position of Vegetative Cell Nucleus (VCN), Sperm Cell Nucleus (SCN) and Cytoplasmic Connection (CC). Scale bars correspond to $5 \mu \mathrm{M}$.

Figure 4: The LARP6C La-module binds the B-type RNA boxes. (A), Consensus sequences of the conserved motifs and their approximate location (add the graph please shared by the 5'-UTRs of LARP6C mRNA targets. Calorimetric (B, D) and EMSA (C, E) analyses of the interaction between the LARP6C La-module (encompassing residues 137-332) and oligos B1, B2 (B, C), $\mathrm{U}_{20}(\mathbf{C}), \mathrm{B} 3$ and B4 (D, E). In (B) and (D): for each graph, the upper panel corresponds to the raw titration data showing the thermal effect of injecting an RNA oligo solution into a calorimetric cell containing the recombinant LARP6C La-module. The lower panels show the normalized heat for the titrations obtained by integrating the raw data and subtracting the heat of the RNA dilution. The red lines on graphs for oligos B1 and B2 (B) represent the best fit derived by a non-linear best-square procedure based on an independent binding site model. The dissociation constants $(\mathrm{Kd})$ are indicated for the B1 and B2 oligos, the thermodynamic parameters are shown in Table S2. (C, E), EMSA analyses of LARP6C La-module binding to: B1, B2 or oligo $U_{20}(C)$, B3 or B4 (E). Decreasing concentrations ( $\mu \mathrm{M})$ (88 (lane 1), 29.3 (lane 2), 9.8 (lane 3), 3.3 (lane 4), 1.1 (lane 5), 0.4 (lane 6), 0.12 (lane 7), 0.04 (lane 8) and 0 (lane 9)) of the recombinant LARP6C La-module were mixed with $3 \mathrm{nM}$ of 5 '-labelled oligos. B stands for Bound, F for Free, blue asterisks mark the RNA-protein complex and the red arrows on panels $E$ shows samples retained into the gel wells. Experiments were conducted in absence (-tRNA) or presence (+tRNA) of unlabelled competitor (tRNAmix of E.coli MRE 600 at 0.01 $\mathrm{mg} / \mathrm{mL}$ concentration). Graphs on panel $\mathrm{C}$ show the quantification of the bound RNA fraction versus the protein concentration in the absence (black lines) or presence (red lines) of tRNA competitor. The values of the dissociation constants are reported. $\mathrm{Kd}$ reported for the EMSA experiments were calculated out of three independent replicates with the following standard deviations: LARP6C-B1 (-tRNA): 0.8 +/- 0.1; 
LARP6C-B1 (+tRNA): 3.5 +/- 0.3; LARP6C-B2 (-tRNA): 1.9 +/- 0.3; LARP6C-B2 (+tRNA): $5.6+/-0.2$.

Figure 5: larp6c loss-of-function does not affect the steady-state transcripts levels of its target in dry pollen. (A), Heat map representation of the $\log _{2}(r p k m)$ values of genes that are differentially expressed between wild-type (WT) and larp6c-3 plants. The heat map was built from the list of DE genes which log2(rpkm) value is between -2 and +10 (2142 genes: $98.5 \%$ of the DE genes).(B), Plot representation of the log2 values of the ratios: (larp6c-3/wild type) (upper panel) and log2(6C-FH/wild type) (lower panel) for the 2174 genes found to be differentially expressed in larp6c-3 mutant pollen. Red lines mark the cut off value $(\log 2(1.5)$ and $\log 2(1 / 1.5))$. (C) Venn diagram representation of the number of transcripts which are DE in larp6c-3 and/or immunoprecipitated by 6C-FH. Note that of the 115 RIP targets, 19 were not present in the transcriptomic data from the RNA-seq. (D) plot representation of the log2(larp6c3/WT) for mRNAs identified by RIP-seq. Red lines mark the cut off value (log2(1.5) and $\log 2(1 / 1.5))$.

Figure 6: LARP6C binding at the 5'UTR reduce translation and increases mRNA levels. (A) Schematic representation of the YFP reporter constructs, (B) RT-qPCR monitoring of YFP mRNA levels. To normalize YFP mRNA levels to transformation efficiency we used the levels of the HPTII mRNA, encoded by the HPTII gene carried by the YFP binary plasmid but not the tRFP-LARP6C one. SDs were calculated from three biological replicates. $p$-value were obtained using a student-t test. ${ }^{* *} P<0.005$, n.s: non significant. (C) Western blot analyses of tRFP-LARP6C and YFP protein levels. Two western blots were prepared and respectively hybridized with anti-LARP6C or GFP antibodies. Levels of UGPase were used as loading control. Representative images of three replicates are shown. (D) Confocal imaging of YFP from leaves not transformed with tRFP-LARP6C (left panels) and of YFP and tRFP-LARP6C distribution (right panels). Scale bars represent $20 \mu \mathrm{M}$. Leaves that were observed are different from those used to prepare total RNA and protein extracts. Representative images of three biological replicates are shown.

Figure 7: The pollen tube guidance defect of larp6c lof mutants is aggravated in the presence of galvestine-1. (A), Monitoring of pollen tube growth in the presence 
of galvestine-1. Whisker boxplot representation of the length of wild-type or larp6c-3 pollen tubes grown in vitro in the presence or absence of galvestine-1. (B), Semi in vivo pollen tube guidance assays. Assays were conducted either in the presence of $1 \%$ DMSO (untreated) or in the presence of $10 \mu \mathrm{M}$ of galvestine-1. Results are represented as whisker boxplots.

Figure 8: A model of LARP6C-B-box-PABP mediated translational silencing in the pollen tube guidance process. (A) In dry pollen grain, LARP6C binds to B-box 5'UTR motif of its mRNA targets and together with the poly $(A)$ Binding Protein (PABP) localises to cytoplasmic aggregates to maintain its targets mRNA in translationally silent state. (B) During pollen tube tip growth, LARP6C could accompany its bound mRNA targets (as shown in human LARP6) and keep them translationally repressed until an external signal (likely ovule-emitted cue) is perceived at the pollen tube tip. Perceived paracrine signal would then relieve LARP6C-mediated repression through a yet unknown process to permit localized translation of factors involved in guided pollen tube growth including localized secretory machinery, receptors and membrane lipids. This is consistent with the observation that the chemical inhibition of MGD2 enzymatic activity aggravates guidance defect but not pollen tube growth. 
A

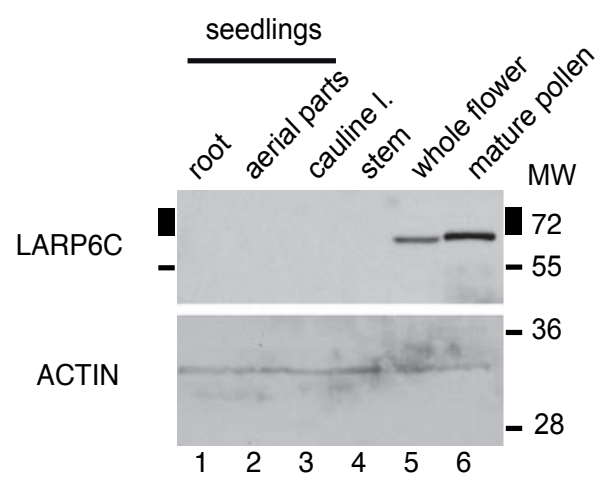

B larp6c-3 larp6c-4
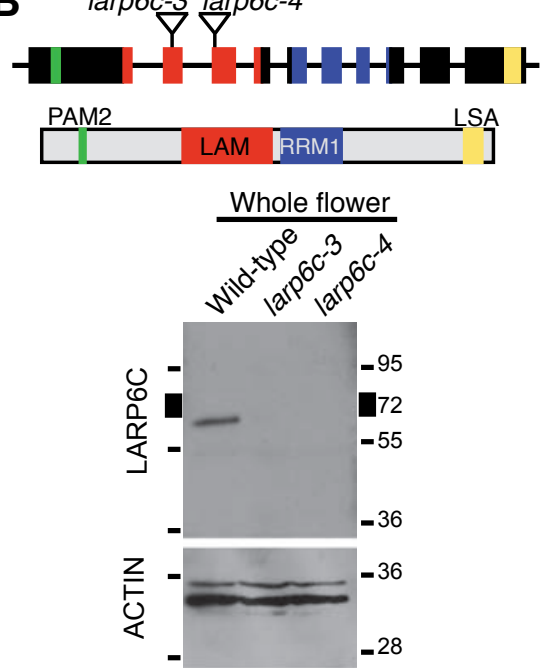

C

\begin{tabular}{ccccccc}
\hline \multicolumn{2}{c}{ Parental Genotypes } & $\begin{array}{c}\text { Progeny } \\
\text { (total no) }\end{array}$ & $\begin{array}{c}\text { No of expected } \\
\text { mutants }\end{array}$ & $\begin{array}{c}\text { No of observed } \\
\text { mutants }\end{array}$ & $\begin{array}{c}\text { Transmission } \\
\text { Efficiency (\%) }\end{array}$ & khi2; p-value \\
\hline Male & Female & & & & & \\
$6 c-3 /+$ & WT & 827 & 413.5 & 352 & 74.1 & $9.14 ;<0.005 \mathrm{~s}$ \\
WT & $6 c-3 /+$ & 641 & 320.5 & 321 & 100 & $8.4 ; \mathrm{ns}$ \\
$6 c-4 /+$ & WT & 1051 & 525.5 & 426 & 68.2 & $18.8 ;<0.001 \mathrm{~s}$ \\
WT & $6 c-4 /+$ & 1147 & 573.5 & 564 & 96.7 & $0.15 ; \mathrm{ns}$ \\
\hline
\end{tabular}

D

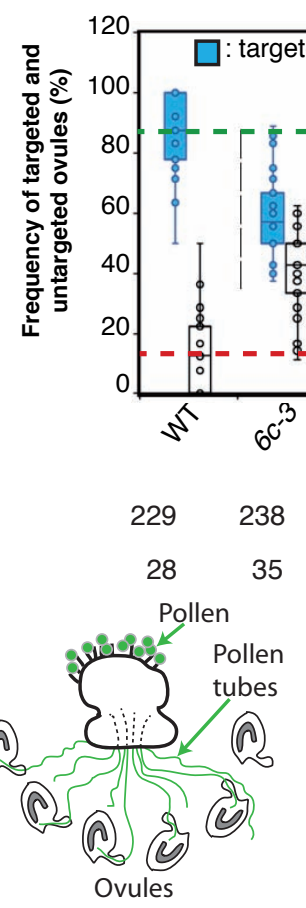

Semi In Vivo

\section{E}

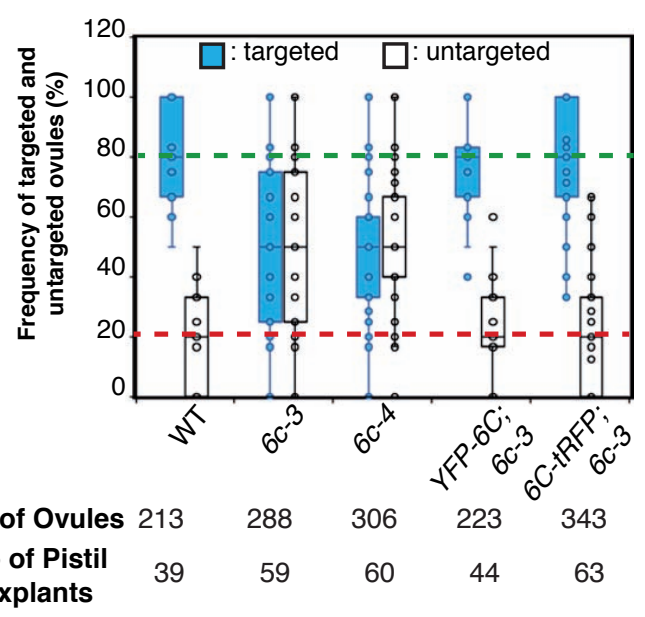

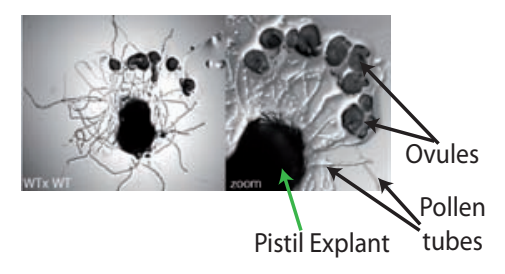

Figure 1

Billey et al. 

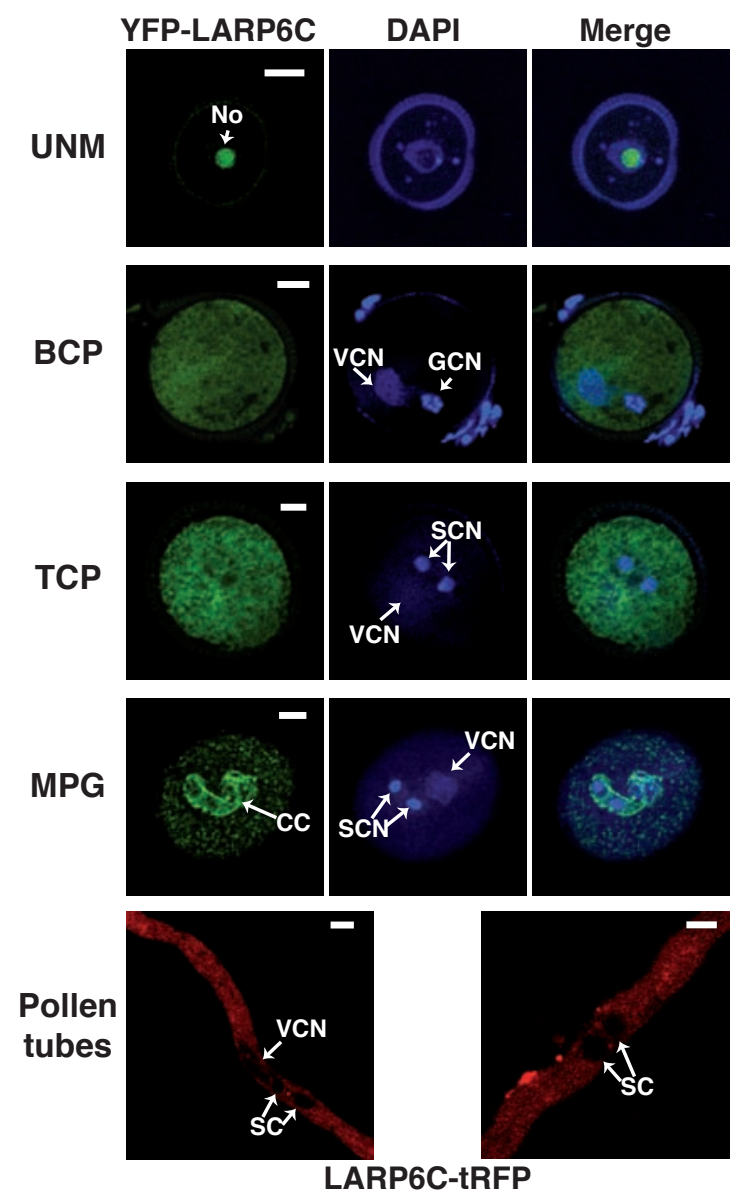

Figure 2

Billey et al. 
bioRxiv preprint doi: https://doi.org/10.1101/2020.11.27.401307; this version posted November 27, 2020. The copyright holder for this preprint (which was not certified by peer review) is the author/funder. All rights reserved. No reuse allowed without permission.

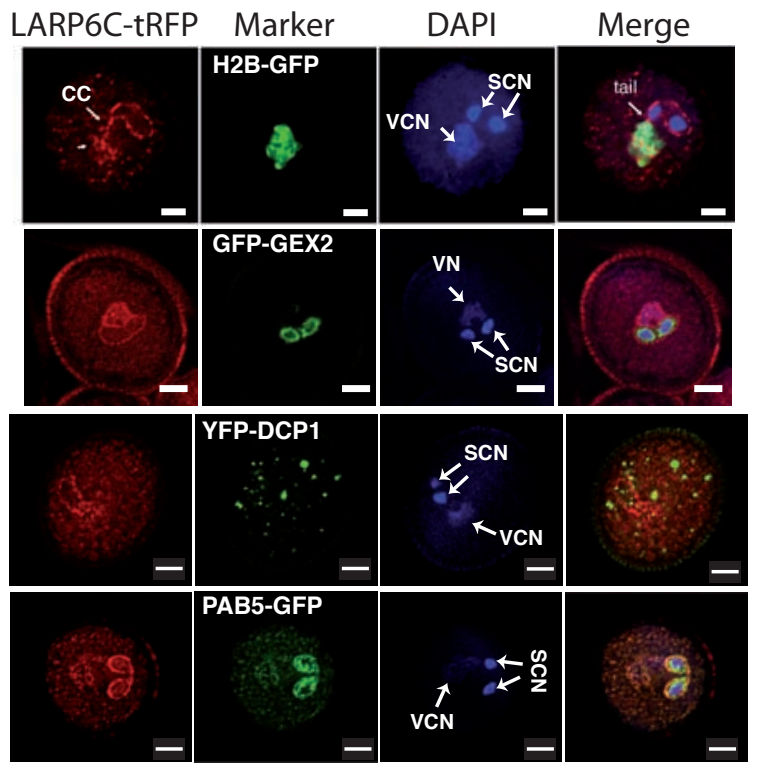

Figure 3

Billey et al. 
A
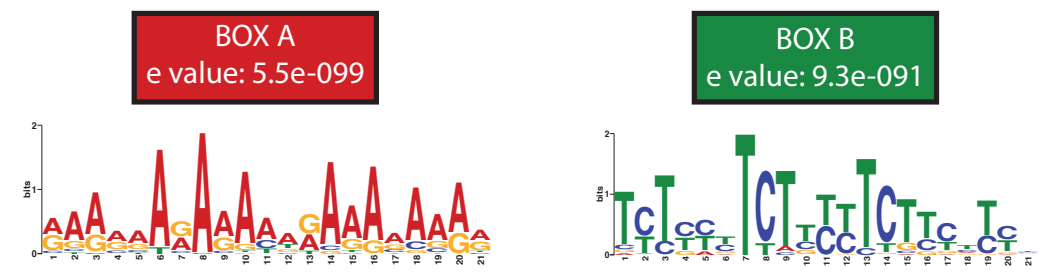

B

B1: 5'-CUUCUUUCUUUCUUUUUUGUU-3' B2: 5'-UCUCUCUGUUUCUUAUUCAUC-3'

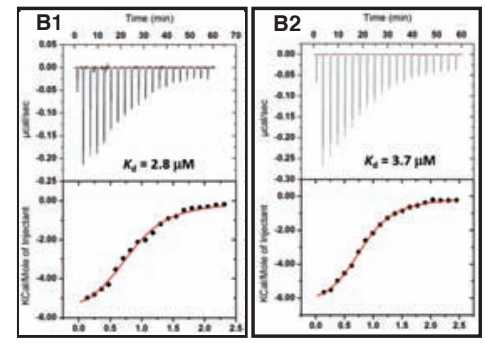

D

B3: 5'-Ccucucccccceucccccuc-3' B4: 5'-CCUCUCUCCUUCUCCUCCCUC-3'

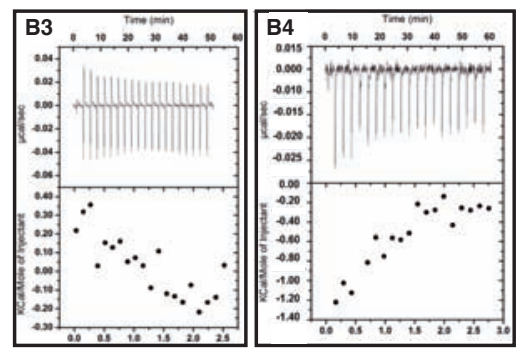

C
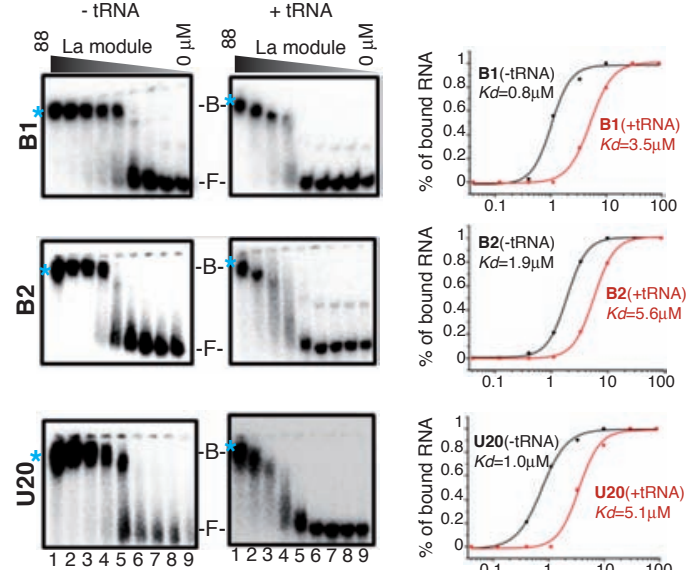

E
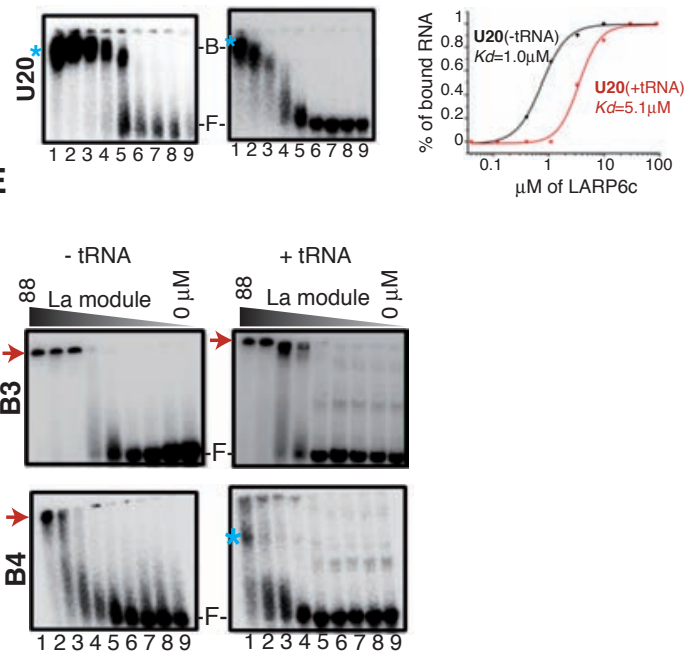

Figure 4

Billey et al. 

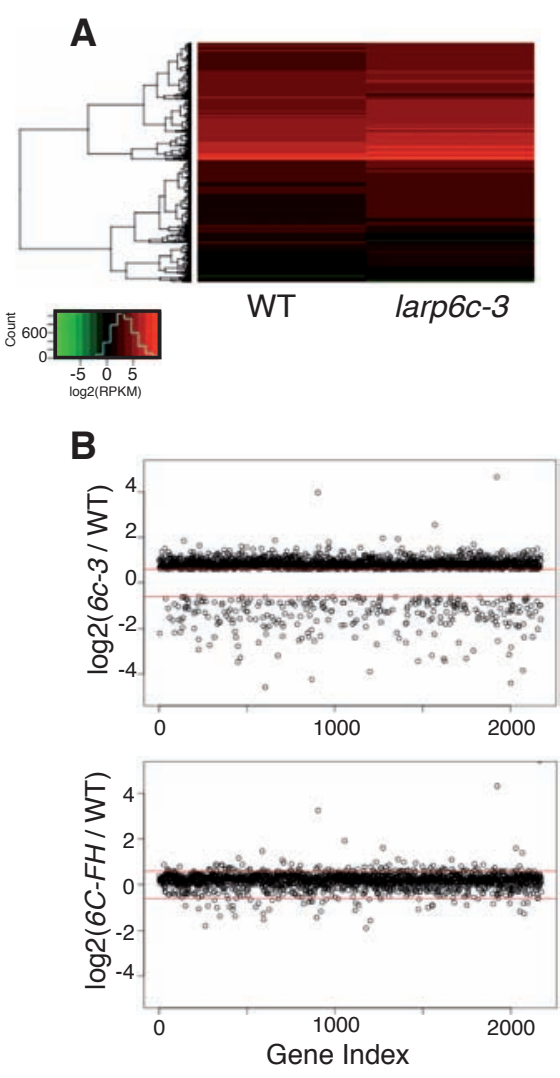

C

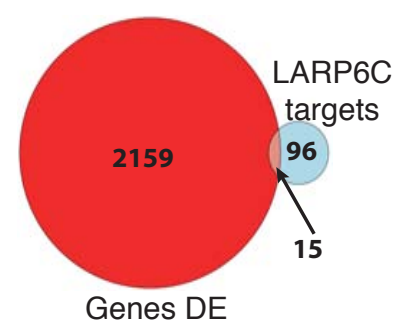

D

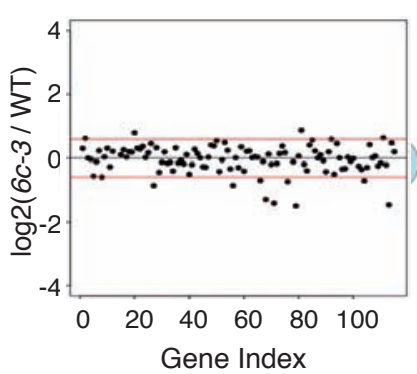

Figure 5

Billey_et al 

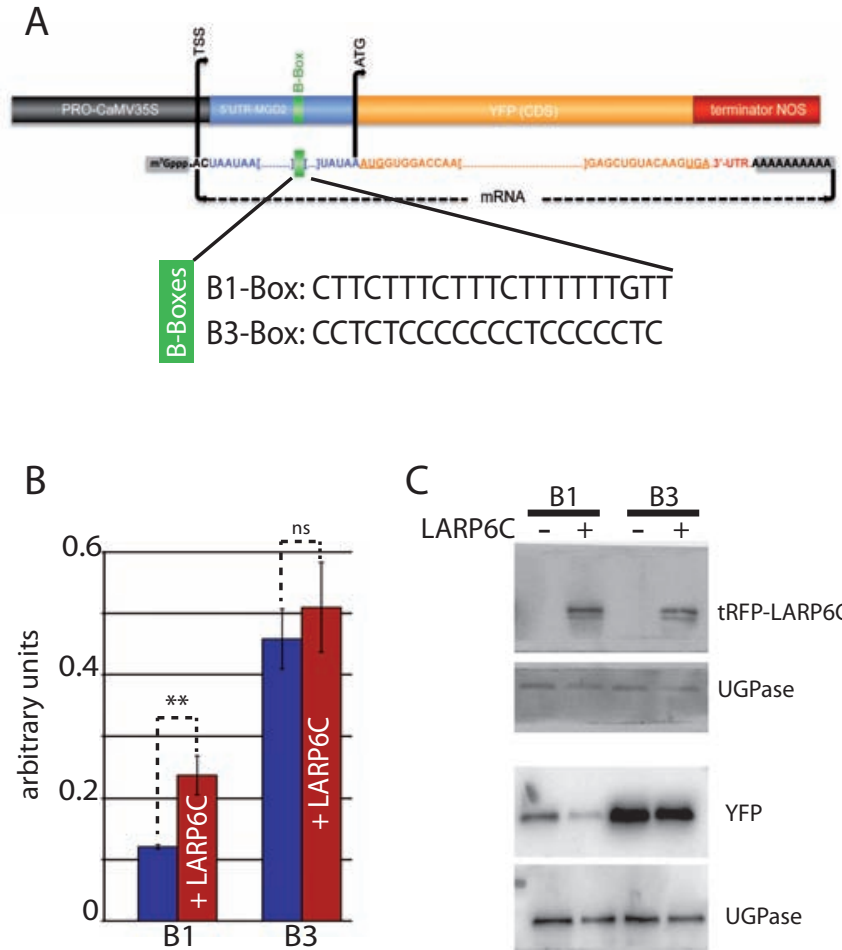

C

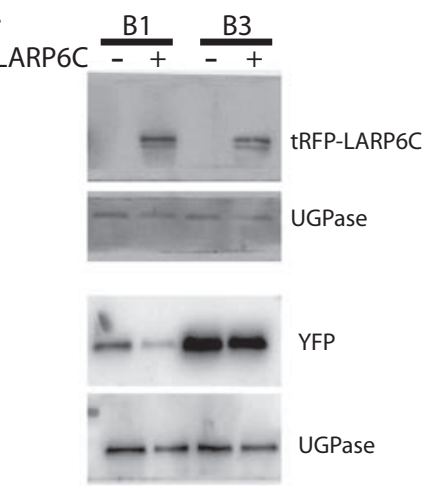

D

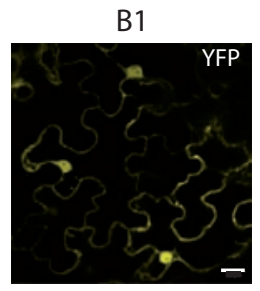

B1 + LARP6C

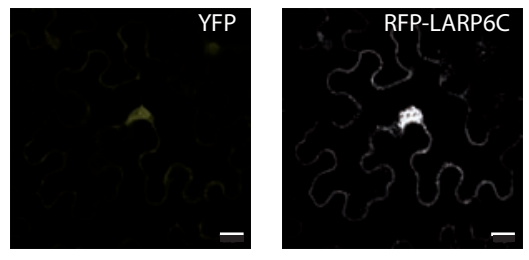

B3

B3 + LARP6C
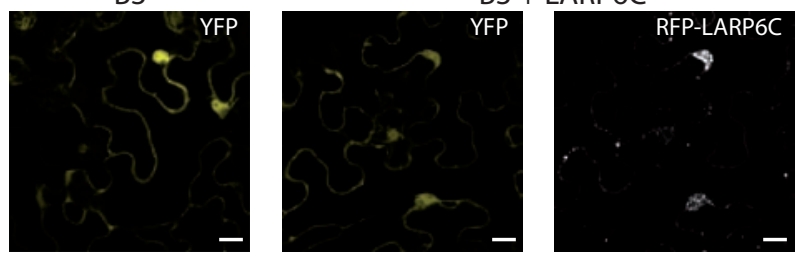

Figure 6

Billey et al. 
A

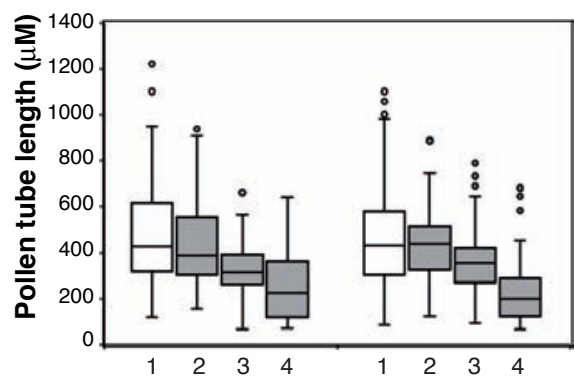

1: untreated

Wild-Type

larp6c-3

2: mock (1\% DMSO)

3: $5 \mu \mathrm{M}$ galvestine-1

4: $10 \mu \mathrm{M}$ galvestine-1

B

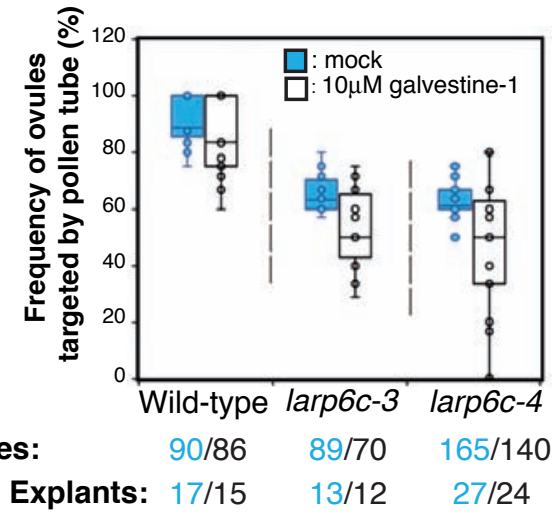

Figure 7

Billey et al. 


\section{A Mature Pollen Grain}
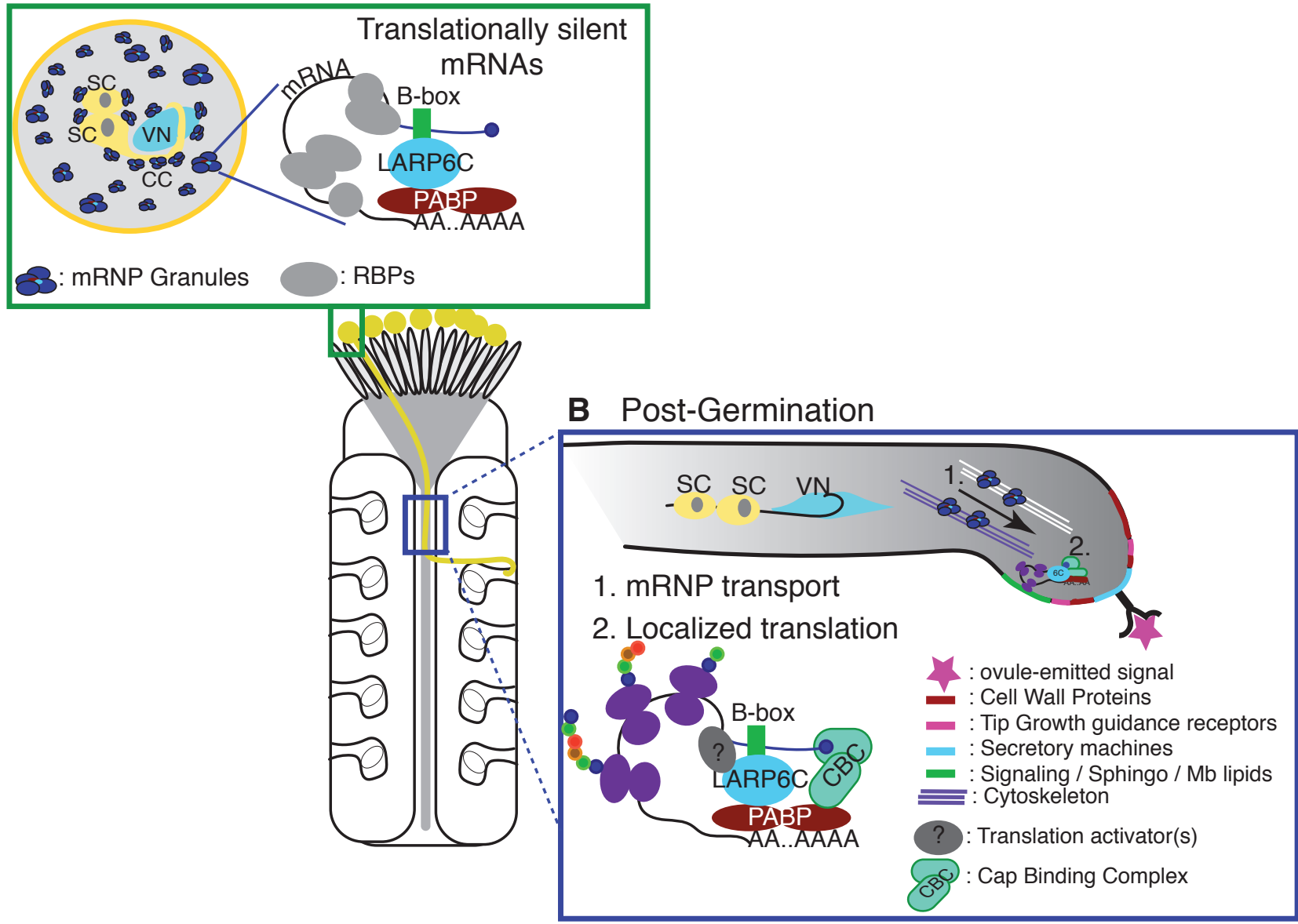

Figure 8, Billey et al. 